\title{
An Image Morphing Technique Based on Optimal Mass Preserving Mapping
}

\author{
Lei Zhu, Yan Yang, Steven Haker, and Allen Tannenbaum
}

\begin{abstract}
Image morphing, or image interpolation in the time domain, deals with the metamorphosis of one image into another. In this paper, a new class of image morphing algorithms is proposed based on the theory of optimal mass transport. The $L^{2}$ mass moving energy functional is modified by adding an intensity penalizing term, in order to reduce the undesired double exposure effect. It is an intensity-based approach and, thus, is parameter free. The optimal warping function is computed using an iterative gradient descent approach. This proposed morphing method is also extended to doubly connected domains using a harmonic parameterization technique, along with finite-element methods.
\end{abstract}

Index Terms-Image interpolation, image morphing, image warping, mass preserving mapping, Monge-Kantorovich flow, optimal transport.

\section{INTRODUCTION}

I MAGE morphing, sometimes referred to as "image interpolation in the time domain," deals with the metamorphosis of one image to another [21]. It is a technique widely used in television commercials, music videos and motion pictures. Image morphing has also been used for facial recognition [37]. Given a pair of images, the goal of image morphing is to find a sequence of intermediate images, such that the first image in the sequence is equal to the first given image (starting image) and the last image is equal to the second given image (ending image). The process begins with finding a reasonable warping function between the two images, and this warping function is then used to interpolate the position of pixels through the in-between sequence. Finally, intensity or color interpolation (i.e., cross dissolving) is performed to generate the intermediate images.

There have been many algorithms proposed for image morphing. Some of the most popular approaches are mesh warping,

Manuscript received September 7, 2006; revised January 22, 2007. This work was supported in part by the National Science Foundation, in part by the Air Force Office of Scientific Research, in part by ARO, in part by MURI, in part by MRI-HEL, and in part by the National Institutes of Health (NIH) under Grant NAC P41 RR-13218 through Brigham and Women's Hospital. This work is part of the National Alliance for Medical Image Computing (NAMIC), funded by the NIH through the NIH Roadmap for Medical Research, Grant U54 EB005149. The associate editor coordinating the review of this manuscript and approving it for publication was Prof. Stanley J. Reeves.

L. Zhu, Y. Yang, and A. Tannenbaum are with the Department of Biomedical Engineering and the School of Electrical and Computer Engineering, Georgia Institute of Technology, Atlanta, GA 30332 USA (e-mail: zlzl@ece.gatech.edu; zhulei1976@hotmail.com; yan.yang@gatech.edu; tannenba@ece.gatech.edu).

S. Haker is with the Surgical Planning Laboratory, Brigham and Women's Hospital and Harvard Medical School, Boston, MA 02115 USA (e-mail: haker@bwh.harvard.edu).

Color versions of one or more of the figures in this paper are available online at http://ieeexplore.ieee.org.

Digital Object Identifier 10.1109/TIP.2007.896637 field warping, and energy-based warping. In mesh warping [35], features are specified by a nonuniform control mesh, and the warping function is usually generated by a spline interpolation. This class of mesh warping algorithm usually shows good distortion behavior, but it has a critical drawback in specifying features since the features on the control mesh may have an arbitrary structure. It is also time consuming to define the feature correspondence via a user interface. In field morphing [2], a pair of corresponding lines is specified. The mapping of a point in the vicinity of a line can be determined by its distance from the line. In the case of multiple line pairs, the warping of a given point is calculated by a weighted sum of mappings of all line pairs. This method is easy to use to specify corresponding features. However, sometimes a part of the image may appear in unrelated regions in the in-between images (often referred to as "ghosts"). Energy minimization-based warpings usually guarantee the one-to-one mapping property, which prevents the warped image from folding back upon itself. For example, in Lee et al.'s work [21], points, polylines, and curves are sampled and reduced to a collection of points. These points are then used to generate the warping function by minimizing an energy functional. A similar method has been applied to facial morphing based on Navier elastic body spline [15]. All of the above approaches fall into the landmark-based category and require user inputs of the corresponding features.

The approach proposed in the present work is intensity based. Here, one uses only information given by the images such as pixel intensities to perform the warping. A successful intensity-based algorithm can achieve automatic morphing without user inputs or prior assumptions on special shapes or features of the objects in the image. See [31] for a review of the literature and an extensive list of references on this subject. We should also mention the nice work of Iwanowski [17] in which an image morphing approach is proposed that combines morphological interpolation and linear filtering and does not require control points or landmarks. In this paper, we present an automatic morphing algorithm using a completely different approach which is based on the theory of optimal mass transport. It is important to note that we do not claim that our method is optimal in any sense for morphing, but does throw some new light on the morphing problem, and seems reasonable for certain types of images in which there is an elastic deformation as demonstrated in some of our experiments given below.

Optimal mass transport problem was first formulated by Gaspar Monge in 1781. It concerned finding an optimal way, in the sense of minimal transportation cost, of moving a pile of soil from one site to another. This problem was given a modern formulation by Kantorovich in 1948 [19], and is also known 
as the Monge-Kantorovich problem (MKP). The optimal mass transport problem has been extensively studied in the fields of econometrics, fluid dynamics, automatic control, transportation, statistical physics, shape optimization, expert systems, and meteorology [26]. Recently, this problem has been studied within the context of content-based image retrieval [22], [27], [28]. Pixels in an image are divided into several bins according to their color and spatial locations. The Earth Mover's distance (EMD) is then calculated between the bins of two images and used for image retrieval.

Our interest in MKP arose from our visualization work in medical applications. For example, a flattened representation of colon surface is helpful for the detection of colon polys [13] and a flattened representation of vessel surface is useful for the study of the correlation between wall shear stress and the development of atherosclerosis [39]. Among various flattening techniques, a mapping that preserves the area is of special interests. In this approach, an angle preserving flattening is first constructed through harmonic analysis, and then the optimal mass preserving mapping is applied in order to correct area distortions. The resulting mapping is an area preserving mapping with minimal distortion. We have also proposed an MKP-based image registration algorithm [14], in which a pseudo-mass (intensity-weighted area) is preserved. Our algorithm can handle area preserving registration [12] naturally by simply assigning the pseudo mass density to unity on the entire domain of the image. Other approaches to using various classes of diffeomorphisms for registration and warping may be found for example in [25], [31], [32], and the references therein.

In this paper, we present an improved approach for image morphing, with special efforts taken to reduce the double exposure effect (also referred to as the "fade-in and fade-out" effect). An improved three-step gradient descent approach (as opposed to the two-step approach used in [14] and [40]) is employed here for rectangular domains. We also explain how to extend this approach to irregular multiconnected domains.

We now outline the contents of this paper. In Section II, we give a brief review of the optimal mass transport problem. In Section III, we present our approach for image morphing between two rectangular images, using the improved gradient descent method. Two types of comparison terms are studied in this section. In Section IV, we extend our morphing algorithm to doubly connected domains, based on a harmonic parameterization technique. In Section V, we illustrate the proposed algorithms using real images as examples. Finally, in Section VI, we summarize the contributions of this paper and discuss possible future directions. An Appendix is provided at the end of the paper to give more mathematical details of our methods.

\section{Monge-Kantorovich Problem}

We now give a modern formulation of the MKP. Assume that $\Omega_{0}$ and $\Omega_{1}$ are two domains in $\mathbf{R}^{d}$ with smooth boundaries, each with a positive density, $\mu_{0}$ and $\mu_{1}$, respectively. Further, we assume that both domains contain same total amount of mass

$$
\int_{\Omega_{0}} \mu_{0}=\int_{\Omega_{1}} \mu_{1}
$$

A mapping $u: \Omega_{0} \rightarrow \Omega_{1}$ is called mass preserving (MP), if $u$ satisfies

$$
\mu_{0}=|D u| \mu_{1} \circ u \text {. }
$$

We denote this as $u \in M P$. Equation (2) is called the Jacobian equation, where $|D u|$ denotes the determinant of the Jacobian of $u$, and o denotes the composition of two functions. Equation (2) implies, for example, that if a small region in $\Omega_{0}$ is mapped onto a larger region in $\Omega_{1}$, there must be a corresponding decrease in density in order for the mass to be preserved.

There exist infinite number of such mappings. A criterion, or a metric, must be defined in order to obtain an optimal mapping. In this work, we employ the $L^{p}$ Kantorovich-Wasserstein metric defined as follows:

$$
d_{p}\left(\mu_{0}, \mu_{1}\right)^{p}:=\inf _{u \in M P} \int\|u(x)-x\|^{p} \mu_{0}(x) d x .
$$

This metric places a penalty on the mass weighted $L^{p}$ distance of each bit of material moved by mapping $u$.

In this paper, we take $p=2$. One can show that in this case one gets a unique minimizer given by the gradient of a convex function; see [3], [11], [20], and the references therein. Note that the Kantorovich-Wasserstein metric defines the distance between two mass densities, by seeking the "cheapest" way to transport the mass from one domain to another with respect to the metric (3). The optimal MP mapping thus defined is symmetric, the optimal mapping from $\Omega_{0}$ to $\Omega_{1}$ being the inverse of the optimal mapping from $\Omega_{1}$ to $\Omega_{0}$.

\section{IMAGE MORPHING ON RECTANGULAR REGIONS}

In the context of image morphing, we can assume that the mass density is the image intensity and directly apply the optimal mass transport algorithm on two related images to generate a deformed grid. The in-between image sequence can be obtained using cross dissolving. However, a mapping that maps a small high intensity region to a large low intensity region is not desirable since it will cause double exposure effect in the in-between images. The $L^{2}$ Kantorovich-Wasserstein metric imposes a penalty only on the work spent on moving mass from one shape to another, but not on the change of mass densities or image intensities. Hence, we add a comparison term to the distance metric (3) to penalize the change of intensity in the image. In this section, we only consider the image morphing problem between two rectangular domains, and leave the discussion of more general domains to Section IV.

The idea is to minimize a functional of the following form over an $L^{2}$ MP mappings $u: \Omega_{0} \rightarrow \Omega_{1}$

$$
M_{\alpha}[u]:=\int C\left(I_{0}, I_{1} \circ u\right) d x+\alpha \int\|u(x)-x\|^{2} \mu_{0}(x) d x
$$

where $\alpha \in \mathbf{R}$ is a fixed positive number. The first term controls the "goodness of fit" between the intensity images $I_{0}: \Omega_{0} \rightarrow$ $\mathbf{R}$ and $I_{1} \circ u: \Omega_{1} \rightarrow \mathbf{R}$, and when $I_{0}$ and $I_{1}$ are identical, this term reaches its minimum. The second Monge-Kantorovich term controls the warping of the map. The function $\mu_{0}$ is the mass density defined on $\Omega_{0}$, which could be the same as $I_{0}$ or a smoothed version of $I_{0} \cdot \mu_{0}$ could also be any scalar field that is appropriate for the underlying physical model. Similarly, $\mu_{1}$ is 
the mass density defined on $\Omega_{1}$. By adjusting $\alpha$, we control the tradeoff between minimal mass transport and minimal intensity change. It must be pointed out that by adding the comparison term $C\left(I_{0}, I_{1} \circ u\right)$, the energy functional (4) may have multiple local minima, and the resulting optimal mapping is no longer a curl-free field as in the classical $L^{2}$ MKP.

We note that the comparison term $C\left(I_{0}, I_{1} \circ u\right)$ may be taken to be any metric that measures the similarity between the transformed source image and the target image, e.g., the sum of squared difference (SSD), likelihood measurement, correlation ratio, normalized correlation, or mutual information (MI) [34]. In this paper, SSD and MI are adopted as the similarity measures, based on the characteristics of our testing images.

If SSD is used as the similarity measure [14], we are minimizing the following energy functional:

$$
M_{\alpha}[u]=\int\left(I_{1} \circ u-I_{0}\right)^{2} d x+\alpha \int\|u(x)-x\|^{2} \mu_{0}(x) d x
$$

and if MI is used as the similarity measure [16], the energy functional has the form

$$
\begin{array}{r}
M_{\alpha}[u]=-\int_{i_{0} \times i_{1}} p_{u}^{I_{0}, I_{1} \circ u}\left(i_{0}, i_{1}\right) \log \frac{p_{u}^{I_{0}, I_{1} \circ u}\left(i_{0}, i_{1}\right)}{p^{I_{0}}\left(i_{0}\right) p_{u}^{I_{1} \circ u}\left(i_{1}\right)} d i_{0} d i_{1} \\
+\alpha \int_{\Omega_{0}}\|u(x)-x\|^{2} \mu_{0}(x) d x .
\end{array}
$$

Note that there is a minus sign before the MI term, since the more similar the two images are, the larger the MI measure is. It should also be pointed out that the first integral is taken over the domain of $i_{0} \times i_{1}$, where $i_{0}$ and $i_{i}$ are intensity levels in the histograms of the two images. The probability density functions $p^{I_{0}}\left(i_{0}\right)$ and $p_{u}^{I_{1} \circ u}\left(i_{1}\right)$ can be estimated by the 1-D nonparametric Parzen-Rozenblatt density model [9]

$$
p^{I_{0}}\left(i_{0}\right)=\frac{1}{V} \int_{\Omega_{0}} \psi_{1 D}\left(I_{0}(x)-i_{0}\right) d x
$$

and

$$
p_{u}^{I_{1} \circ u}\left(i_{1}\right)=\frac{1}{V} \int_{\Omega_{0}} \psi_{1 D}\left(I_{1}\left(u(x)-i_{1}\right) d x\right.
$$

where $\psi_{1 D}$ is a 1-D Gaussian window, whose standard deviation can be chosen to be $10 \%$ of the standard deviation of $i_{0}$ and $i_{1}$ for (7) and (8), respectively. $V$ is the area of $\Omega_{0}$, or the number of pixels inside $\Omega_{0}$ for the discrete case. From another point of view, this Parzen-window method is equivalent to smoothing the image histogram using a Gaussian filter.

In a similar way, $p_{u}^{I_{0}, I_{1} \circ u}\left(i_{0}, i_{1}\right)$ can be estimated using a 2-D nonparametric Parzen-Rozenblatt density model as follows:

$$
p_{u}^{I_{0}, I_{1} \circ u}\left(i_{0}, i_{1}\right)=\frac{1}{V} \int_{\Omega_{0}} \psi_{2 D}\left(I_{0}(x)-i_{0}, I_{1}(u(x))-i_{1}\right) d x
$$

where $\psi_{2 D}$ is a 2-D Gaussian window, whose covariance is decided by the covariance matrix of the paired random variables $\left(i_{0}, i_{1}\right)$. For simplicity, we write $\psi_{2 D}$ as $\psi$ in the remaining part of this paper.
There have been a number of algorithms considered for the computation of an optimal transport map. For example, methods have been proposed based on Lagrangian mechanics that are closely related to the ideas in fluid dynamics [3], and geometric methods have also been employed as in Culler-Purser [7]. Perhaps the most common approach is to reduce the $L^{2}$ optimal transport problem to a linear programming problem [26]. However, a fundamental difficulty of linear programming is the computational complexity [18].

We propose to use a different approach to solve the modified optimal mass transport problem (4). The basic idea is to start from an initial MP mapping $u^{0}$ from $\Omega_{0}$ to $\Omega_{1}$, and based on the fact that the composition of two MP mappings is still an MP mapping, the second step is to update the mapping iteratively in a gradient descent way using MP mapping $s^{-1}$ that maps $\Omega_{0}$ onto itself and satisfies the MP property to decrease the pure $L^{2}$ MKP energy functional without the comparison term. It can be proved that the curl of the mapping is also decreasing in this process [1]. The resulting MP mapping $\tilde{u}$ is a curl-free field. Finally, we start from this curl-free field $\tilde{u}$ and use a similar gradient descent approach to minimize the modified energy functional (4). As we mentioned earlier, the functional (4) may have multiple local minima. Starting with a curl-free MP mapping $\tilde{u}$ will make the final MP mapping $u^{\infty}$ look more natural by putting most curl into the dark region of the images, as shown in the results described in Section V.

\section{A. The Initial MP Mapping $u^{0}$}

For arbitrary domains, the initial mapping can be solved using the method proposed by Dacorogna and Moser [8]. Since we are working on more regular domains (specifically, two rectangular domains in 2-D or two cubical domains in 3-D), a simpler algorithm is implemented here. To further simplify the problem, we assume we are working in $\mathbf{R}^{2}$ with $\Omega_{0}=\Omega_{1}=[0,1]^{2}$, the generalization to higher dimensions being straightforward. The idea of the proposed method is that we solve a 1-D mass transport problem in one direction and then solve a family of 1-D mass transport problems in the other direction. In 1-D, the optimal transport map can then be found by simple quadrature.

We can let the mass first be transported along the lines parallel to the $x$ axis, and then transported along the lines parallel to the $y$ axis. Accordingly, we assume that the initial MP mapping has the form $u^{0}=(a(x), b(x, y))$, where function $a=a(x)$ is defined by equation

$$
\int_{0}^{a(x)} \int_{0}^{1} \mu_{1}(\eta, \rho) d \eta d \rho=\int_{0}^{x} \int_{0}^{1} \mu_{0}(\eta, \rho) d \eta d \rho .
$$

By differentiating (10) with respect to $x$, we have

$$
a^{\prime}(x) \int_{0}^{1} \mu_{1}(a(x), \rho) d \rho=\int_{0}^{1} \mu_{0}(x, \rho) d \rho .
$$

We may now define function $b=b(x, y)$ by equation

$$
a^{\prime}(x) \int_{0}^{b(x, y)} \mu_{1}(a(x), \rho) d \rho=\int_{0}^{y} \mu_{0}(x, \rho) d \rho .
$$


Since $a_{y}=0,|D u|=a_{x} b_{y}=a^{\prime}(x) b_{y}(x, y)$, by differentiating (12) with respect to $y$, we get

$$
\begin{aligned}
a^{\prime}(x) b_{y}(x, y) \mu_{1}(a(x), b(x, y)) & =\mu_{0}(x, y) \\
\left|D u^{0}\right| \mu_{1} \circ u^{0} & =\mu_{0}
\end{aligned}
$$

which is the MP condition. In practice, $a(x)$ and $b(x, y)$ can be found using numerical integration techniques. Given our assumption that $\mu_{0}$ and $\mu_{1}$ are positive everywhere, $a(x)$ is a monotonically increasing function. $b(x, y)$ is also monotonically increasing with respect to $y$ from (12), given that $a^{\prime}(x)$ is always positive. Hence, there is no space folding problem with the initial mapping $u^{0}$.

\section{B. Curl-free MP Mapping $\tilde{u}$}

Once an initial MP mapping $u^{0}$ is found, we need to discover the curl-free MP mapping $\tilde{u}$ by finding the polar factorization [11], [23] of $u^{0}$. Rather than finding $\tilde{u}$ directly, we solve an energy minimizing problem iteratively via a gradient descent method. If the energy functional is defined by the $L^{2}$ Kantorovich-Wasserstein metric, this process is equivalent to finding the polar factorization and is guaranteed to converge to a global optimum [1]. We give the key steps of this method in this section, and more mathematical details can be found in [1], [14], and in the Appendix.

Assume that $u^{0}$ is updated by an MP mapping $s$ from $\Omega_{0}$ to itself, i.e., $u=u^{0} \circ s^{-1}$. Based on the facts that the inverse of an MP mapping is an MP mapping and the composition of two MP mappings is an MP mapping, $u$ is also an MP mapping from $\Omega_{0}$ to $\Omega_{1}$. We take $s$ to be a function of "time" (i.e., gradient descent parameter), initially being the identity map. In order to maintain the MP property, the update of $s$ should have the following form:

$$
s_{t}=\left(\frac{1}{\mu_{0}} \zeta\right) \circ s
$$

for some vector field $\zeta$ on $\Omega_{0}$, with $\operatorname{div}(\zeta)=0$ and $\langle\zeta, \vec{n}\rangle=0$ on $\partial \Omega_{0}, \vec{n}$ being the normal to the boundary of $\Omega_{0}$. Accordingly, $u_{t}$ should satisfy

$$
u_{t}=-\frac{1}{\mu_{0}} D u \zeta
$$

In the gradient descent approach, we take the derivative of the pure $L^{2}$ MKP energy functional with respect to $t$. It can be proved that in 2-D, $\zeta$ should satisfy $\zeta=\nabla^{\perp} f$, where $\perp$ represents a rotation by $\pi / 2$ counter clockwise and $f$ is given by

$$
\begin{aligned}
\triangle f & =-2 \operatorname{div}\left[(u-\underline{i d})^{\perp}\right] \\
f & =0 \text { on } \partial \Omega_{0} .
\end{aligned}
$$

Here, $\underline{i d}$ indicates an identity mapping. Considering (15) and $\zeta=\nabla^{\perp} f$, the updating equation for $u$ is given by

$$
u_{t}=\frac{2}{\mu_{0}} D u \nabla^{\perp} \Delta^{-1} \operatorname{div}\left[(u-\underline{i d})^{\perp}\right]
$$

where $\Delta^{-1}$ stands for the solution to the Poisson equation (16). When the algorithm converges, we have a curl-free vector field $\tilde{u}$, which will serve as the initial mapping for the next step.

\section{C. inimizer $u^{\infty}$}

We use a similar gradient descent approach to find a minimizer $u^{\infty}$ to the functional (4). As above, the idea is to modify an initial mapping $u^{0}$ by an MP mapping from $\Omega_{0}$ to itself in order to decrease the energy (4).

More specifically, the updating equation is given by

$$
u_{t}=\frac{1}{\mu_{0}} D u \nabla^{\perp} \triangle^{-1} \operatorname{div}\left(P^{\perp}\right) .
$$

If SSD is used as the comparison term, $P$ has the form of

$$
P_{\mathrm{SSD}}=\frac{1}{\mu_{0}^{2}}\left(I_{1} \circ u-I_{0}\right)^{2} \nabla \mu_{0}+\frac{2}{\mu_{0}}\left(I_{1} \circ u-I_{0}\right) \nabla I_{0}+2 \alpha(u-\underline{i d})
$$

and if $\mathrm{MI}$ is used as the comparison term, $P$ is given by

$$
\begin{aligned}
P_{M I}=-\frac{1}{V}[ & \left(1+\log \frac{p_{u}^{I_{0}, I_{1} \circ u}}{p^{I_{0}} p^{I_{1} \circ u}}\right) \\
& * \psi_{\alpha}\left(I_{0}(x), I_{1} \circ u(x)\right) \frac{\nabla I_{0}(x)}{\mu_{0}(x)} \\
& -\left(1+\log \frac{p_{u}^{I_{0}, I_{1} \circ u}}{p^{I_{0}} p^{I_{1} \circ u}}\right) \\
& \left.* \psi\left(I_{0}(x), I_{1} \circ u(x)\right) \frac{\nabla \mu_{0}(x)}{\mu_{0}^{2}(x)}\right] \\
+ & 2 \alpha(u-\underline{i d})
\end{aligned}
$$

where $*$ stands for 2-D convolution, and $\psi_{\alpha}$ is the derivative of $\psi$ with respect to its first variable.

Once the algorithm converges, we have the final warping function $u^{\infty}$. Then a cross-dissolving method is performed to generate a sequence of in-between images $I(t)$, such that $I(0)=I_{0}$ and $I(1)=I_{1}$. It is assumed that when time $t$ varies from 0 to 1 , the starting image $I_{0}$ continuously changes to the ending image $I_{1}$. We further require that the same transition rate is applied to all points on the in-between images [21]. Hence, the image warping map at any time $t(t \in[0,1])$ is simply given by

$$
X^{t}(x)=(1-t) x+t u^{\infty}(x)
$$

and the corresponding cross-dissolved image at time $t$ is given by

$$
I^{t}\left(X^{t}(x)\right)=(1-t) I_{0}(x)+t I_{1}\left(u^{\infty}(x)\right) .
$$

$I_{0}$ and $I_{1}$ can also be color images and (22) can be applied to three color components individually. The warping function (21) guarantees the continuous transformation from the source image to the target image, $t$ being the transition rate. One can also guarantee that the intermediate frames are mass preserving simply by shading the pixels in the in-between images according to $\left|D\left(X^{t}\right)^{-1}\right| \mu_{0} \circ\left(X^{t}\right)^{-1}$.

\section{Implementation}

As a summary, our proposed algorithm takes the following steps.

1) Construct an initial MP mapping $u^{0}$ using (10) and (12). 
2) Starting with $u=u^{0}$, update the MP mapping iteratively using (17) and obtain $\tilde{u}$.

3) Starting with $u=\tilde{u}$, update the MP mapping iteratively using (18).

4) Calculate in-between images using the cross-dissolving method by (21) and (22).

An upwinding scheme is used when computing $D u$ in (17), and all other spacial derivatives (including $\nabla^{\perp}$ and div) are computed using standard central differences. Matlab solver poicalc is used to solve the Poisson equation (16). The time step $d t$ can be chosen to be less than

$$
\min _{x, i}\left|\frac{1}{\mu_{0}}\left(\nabla^{\perp} \Delta^{-1} \operatorname{div}\left(P^{\perp}\right)\right)_{i}\right|^{-1}
$$

for the nonlocal flow, where $i$ stands for the component of the vector. The curl-free mapping $\tilde{u}$ is obtained as $t \rightarrow \infty$. In practice, the procedure iterates until the mean absolute curl is sufficiently small. More details about the implementation of Monge-Kantorovich flows may be found in [14].

The numerical implementation of (18) is exactly the same as that for solving $\tilde{u}$ using (17), except $2(u-\underline{i d})$ is substituted by $P$. The iteration stops when the energy functional (4) decreases sufficiently slowly. The optimal mapping $u^{\infty}$ is obtained when the iteration stops.

\section{IMAGe MorPhing ON DOUbly CONNECTED REGIONS}

In the previous section, we presented an MP morphing algorithm between two simply connected domains, or more specifically, two rectangular regions. It is assumed that mass is preserved over the entire domain. In some images, however, the MP condition is valid only between parts of the two images rather than the entire domain. For example, if we are given a sequence of magnetic resonance (MR) images of a human heart taken at different times in the cardiac cycle at the same spatial location, the MP condition is only valid in the myocardium region, but invalid in the heart ventricles since the volume of the blood varies from time to time. A natural approach would be to perform image segmentation first to separate the myocardium and the ventricles, and then perform image morphing only on the myocardial region of interest.

In this section, we extend the MP mapping algorithm to doubly connected domains [41]. The main difficulty comes from the construction of the initial MP mapping. We propose an algorithm that finds $u^{0}$ by using harmonic parameterization. In this approach, the two domains are first harmonically parameterized, and then $u^{0}$ can be obtained in the harmonic coordinate system. A finite-element method (FEM) is applied in conjunction with the gradient descent method to account for the irregularity of the domains.

\section{A. Harmonic Parameterization}

We first show how to construct an analytic function $f^{h}=$ $f_{1}^{h}+i f_{2}^{h}$ for the harmonic parameterization of a doubly connected domain (here $i$ denotes a square root of -1 ). Similar techniques have been applied for colon surface visualization

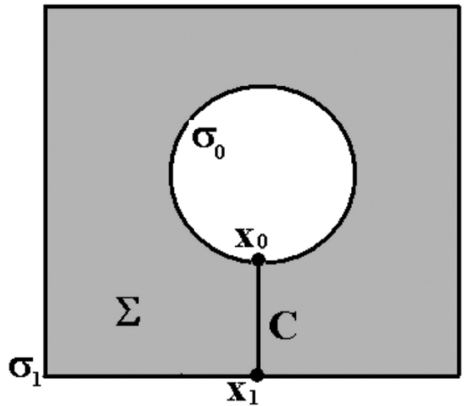

Fig. 1. Dubly onnected domain $\Sigma$ with inner boundary $\sigma_{0}$ and outter boundary $\sigma_{1}$

[13], tissue thickness measurement [36], and defining orthogonal curves for template matching [30].

Assume we have a doubly connected domain $\Sigma$, which has two boundaries: the inner boundary $\sigma_{0}$ and the outer boundary $\sigma_{1}$, as shown in Fig. 1.

The real component of $f^{h}$ is given by

$$
\begin{aligned}
\triangle f_{1}^{h} & =0 \\
\text { with } f_{1}^{h}\left(\sigma_{0}\right) & =0 \quad \text { and } \quad f_{1}^{h}\left(\sigma_{1}\right)=1 .
\end{aligned}
$$

A cut $C$ is then defined from $\sigma_{1}$ to $\sigma_{0}$ starting from an arbitrary point $x_{1}$ on $\sigma_{1}$ along the negative gradient direction of $f_{1}^{h}$ and meet the inner boundary $\sigma_{0}$ at $x_{0}$. Cut $C, \sigma_{0}$ and $\sigma_{1}$ form a new closed and oriented boundary $B$ of domain $\Sigma$

$$
B: x_{0} \stackrel{\sigma_{0}}{\rightarrow} x_{0} \stackrel{-C}{\rightarrow} x_{1} \stackrel{\sigma_{1}}{\rightarrow} x_{1} \stackrel{C}{\rightarrow} x_{0}
$$

with the constraint that $\Sigma$ is on the left hand side of $B$. The boundary condition of the imaginary component $f_{2}^{h}$ on $B$ is given by

$$
f_{2}^{h}(\zeta)=\int_{\zeta_{0}}^{\zeta} \frac{\partial f_{2}^{h}}{\partial s} d s=\int_{\zeta_{0}}^{\zeta} \frac{\partial f_{1}^{h}}{\partial n} d s
$$

according to the Cauchy-Riemann equations. $\zeta_{0}$ is any given point on $B$ that satisfies $f_{2}^{h}\left(\zeta_{0}\right)=0$.

Finally, another Laplace equation

$$
\triangle f_{2}^{h}=0
$$

is solved to give the value of $f_{2}^{h}$ inside domain $\Sigma$.

Numerically, we are working on a triangulated domain and the Laplace equations are solved by a standard FEM technique as we used for colon visualization [13]. Once the analytic function $f^{h}$ is obtained, a curvilinear harmonic polar coordinate system can be defined by taking $f_{1}^{h}$ as one coordinate and $f_{2}^{h}$ as another. $f_{1}^{h}$ can be thought of as a curvilinear "radius" and $f_{2}^{h}$ as the "angle". By scaling $f_{1}^{h}$ and $f_{2}^{h}$ using a constant, we can make $f_{2}^{h}$ run from 0 to $2 \pi$. Thus, the annulus domain $\Sigma$ is mapped onto a rectangular region in an angle preserving manner. Fig. 11 shows such a parameterization on a MR heart image with the ventricle area excluded. 


\section{B. Optimal Mapping Between Doubly Connected Domains}

We follow exactly the same steps as we used for rectangular domains to find the optimal mapping. The first step is to construct an initial MP mapping $u^{0}$. By applying harmonic parameterization, $\left(\Omega_{0}, \mu_{0}\right)$ is mapped onto a rectangle region $\left(\Omega_{0}^{h}, \mu_{0}^{h}\right)$ via conformal mapping $f^{h}=f_{1}^{h}+i f_{2}^{h}$ in an angle-preserving manner. If we define $\mu_{0}^{h}$ as

$$
\mu_{0}^{h}=\left|D f^{h}\right|^{-1} \mu_{0}
$$

the mapping from $\Omega_{0}$ to $\Omega_{0}^{h}$ is a MP mapping. Similarly, domain $\left(\Omega_{1}, \mu_{1}\right)$ is mapped onto another rectangular region $\left(\Omega_{1}^{h}, \mu_{1}^{h}\right)$ via $g^{h}=g_{1}^{h}+i g_{2}^{h}$, where $\mu_{1}^{h}$ is

$$
\mu_{1}^{h}=\left|D g^{h}\right|^{-1} \mu_{1}
$$

Since $\Omega_{0}^{h}$ and $\Omega_{1}^{h}$ are both rectangular regions after the harmonic parameterization, it is easy to define an MP mapping $u_{\text {init }}$ between them using the method presented in Section III-A.

The whole process can be illustrated by the diagram in the equation shown at the bottom of the page.

The resulting initial mapping $u^{0}$ between $\Omega_{0}$ and $\Omega_{1}$ is a composition of $f^{h}, u_{\text {init }}$ and $\left(g^{h}\right)^{-1}$, such that

$$
u^{0}=\left(g^{h}\right)^{-1} \circ u_{\text {init }} \circ f^{h} .
$$

Obviously, $u^{0}$ is an MP mapping since $f^{h}, u_{\text {init }}$ and $\left(g^{h}\right)^{-1}$ are all MP mappings.

Next, we evolve $u^{0}$ according to (17) and then (18) to find the optimal mapping $u^{\infty}$. Due to the irregularity of the domains, an FEM method is used to solve the Poisson equation (16) on a triangulated surface. An upwind scheme is applied for computing $D u$. For all other derivatives, we use a least mean squares (LMS) method to numerically calculate the first-order spatial derivatives. For example, assume that a given point $\left(x_{0}, y_{0}\right)$ has $N$ neighbors $\left(x_{i}, y_{i}\right), i=1 \ldots N$, and a function $\Phi$ is defined such that $\Phi\left(x_{i}, y_{i}\right)=\Phi_{i}$ for $i=0 \ldots N$, it is easy to see that the derivatives of $\Phi$ should satisfy

$$
\left(\begin{array}{c}
\Phi_{x} \\
\Phi_{y}
\end{array}\right)=\left(A^{T} A\right)^{-1} A^{T}\left(\begin{array}{c}
\Phi_{1}-\Phi_{0} \\
\ldots \\
\Phi_{N}-\Phi_{0}
\end{array}\right)
$$

where $A$ is the position difference matrix given by

$$
A=\left(\begin{array}{c}
x_{1}-x_{0}, y_{1}-y_{0} \\
\ldots \\
x_{N}-x_{0}, y_{N}-y_{0}
\end{array}\right) .
$$

When applying harmonic parameterization, different selection of cutting point $x_{1}$ may lead to different initial mapping $u^{0}$. However, the gradient descent method allows every point on the boundary to move along the boundary. Each point will

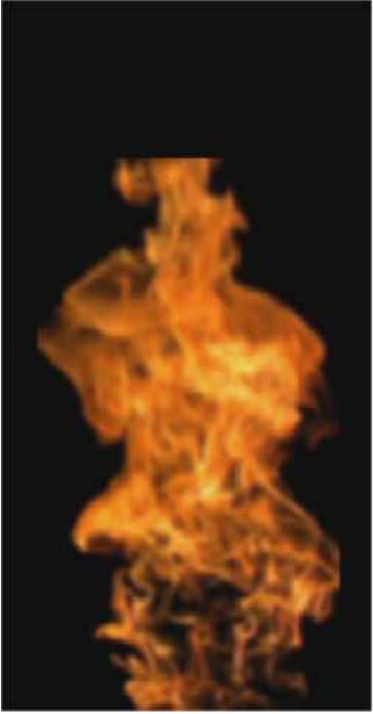

(a)

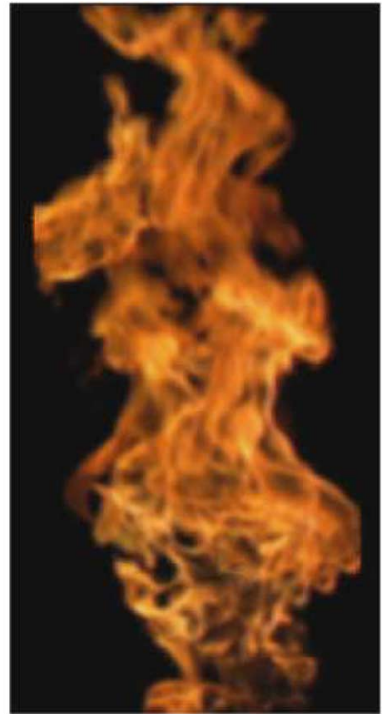

(b)
Fig. 2. Two given flame images. (a) Starting image; (b) ending image.

arrive at its optimal position eventually no matter how it is positioned initially.

\section{RESULTS}

In this section, we show the results of applying the proposed methods to various types of imagery. We first test the modified MP morphing with SSD and MI as the comparison terms on images of fire flames. The second example is an ocean wave video. By selecting several key frames from the video, we interpolate the in-between frames using our proposed method and generate a continuous video. Our method seems well-suited to these applications because the images lack obvious landmarks, require nonrigid mappings for good registration. Further, the intensity-based mass-preservation constraint is reasonable as it reflects physical reality. Registration and interpolation of smoke, clouds, flames and fluids is an active area of research in the computer graphics and image processing community. See [10], [24], and [33], for example, for related methods.

This can serve as an image compression method by storing the selected frames and generating the omitted frames on-the-fly when the video is played. Our extension of the MP morphing algorithm to doubly connected domains is tested over two MR heart images acquired at the systolic and diastolic phases of the cardiac cycle. By applying the proposed method to the images with heart ventricle regions excluded, we are able to generate a natural heart beat through the cardiac cycle using only two given images. The results as images are listed in this paper, and the results as videos can be found on our webpage at

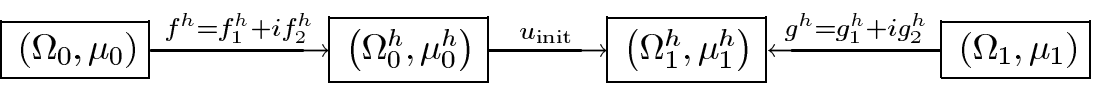




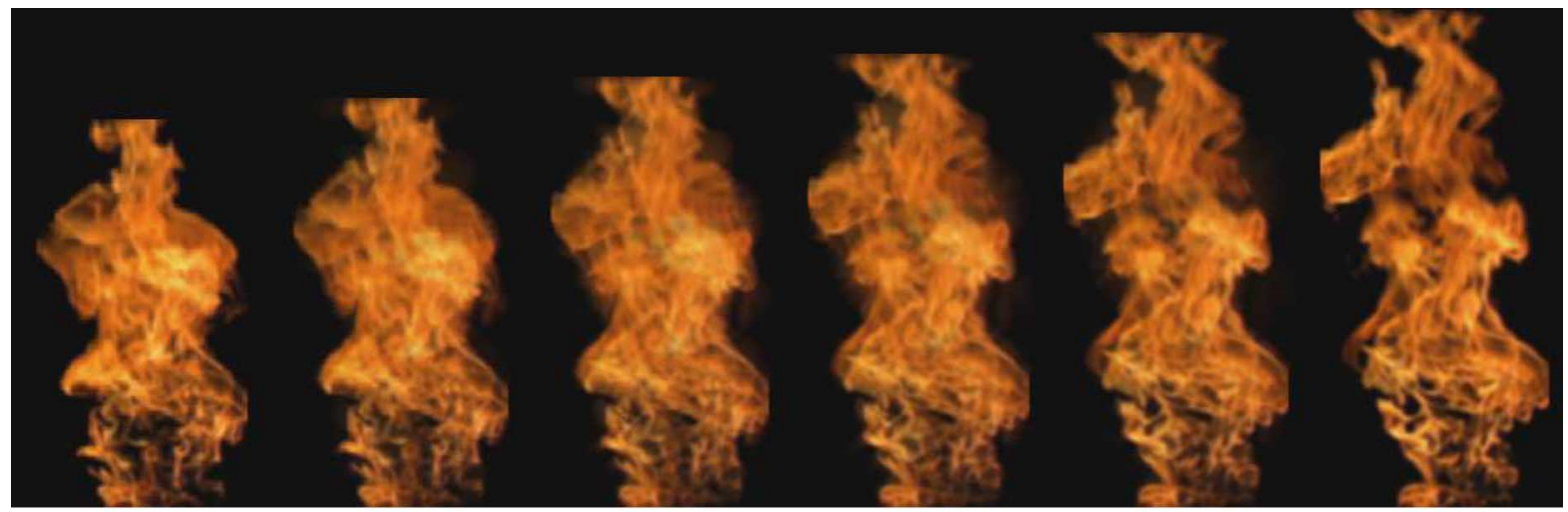

$(\mathrm{t}=0,0.2,0.4,0.6,0.8$ and 1$)$

Fig. 3. Morphing sequence of the flame imagery without comparison terms.

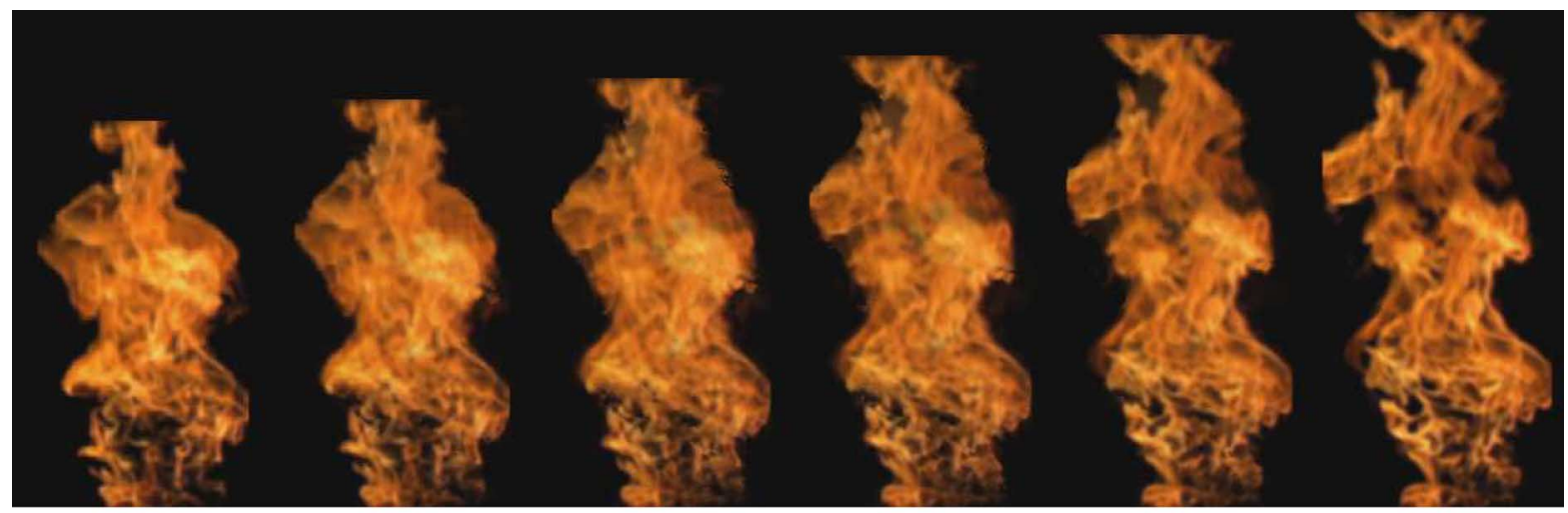

$(\mathrm{t}=0,0.2,0.4,0.6,0.8$ and 1$)$

Fig. 4. Morphing sequence of the flame imagery using SSD as the comparison term.

http://www.bme.gatech.edu/groups/minerva/publications/papers/zhu-extra-index.html.

We now explain the results in more detail. The first example is image morphing between two flame images. The starting image [Fig. 2(a)] and the ending image [Fig. 2(b)] are from a flame video sequence in the Artbeats Digital Film Library (http://www.artbeats.com). The two images are the 24th and the 29th frames in the video. Fig. 3 shows the result of applying pure $L^{2}$ MKP without a comparison term. Figs. 4 and 5 are the results of using SSD and MI as the comparison term, respectively. Fig. 6 compares the results generated by the three different methods at time $t=0.5$ (the starting frame is at $t=0$ and the ending frame is at $t=1$ ). In the pure $L^{2}$ MKP result [Fig. 6(a)], the double exposure effect is obvious. By adding SSD and MI as the comparison term, the double exposure effect has been reduced effectively as shown in Fig. 6(b) and (c). The results of SSD and MI are very similar, however, since both of the image frames come from the same imaging modality. MI has greater flexibility in handling the case where the two image frames come from different imaging modalities, such as PET and MRI in medical imaging, but a better function is needed to be defined to relate the image pixel intensity with the mass in each of the modalities, which is beyond the scope of this paper.
As we have mentioned earlier, adding a comparison term in the energy functional leads to the presence of curl in the final deformed grids. Although SSD is able to reduce the double exposure effect slightly better than MI, it causes broken effects at some edges between the bright and the dark regions due to the high value of curl remaining in these regions. Hence, there is a tradeoff between reducing the double exposure effect and reducing curl. In our previous two-step approach [14], [40], we evolved $u$ directly from $u^{0}$ according to energy functional (4). As a result, the remaining curl is mostly in the "bright" region of the image and it causes unnatural effects as a result. In this work, by starting with a curl-free mapping $\tilde{u}$, the algorithm makes corrections mainly close to the edges of high intensity regions and low intensity regions. Since less energy is required to move pixels within low density regions, the remaining curl is mostly in the "dark" region of the image and it is almost unnoticeable. These effects are more obvious when observed dynamically in videos. Fig. 7 shows the deformed grids for these three cases.

In the second example, a few frames are selected from an ocean wave video, with about 300-ms intervals between two consecutive frames. These frames are used as key frames for image morphing. Our algorithm is applied to two consecutive key frames to interpolate the motion of the wave. In this example, SSD is used as the comparison term. The images in the 


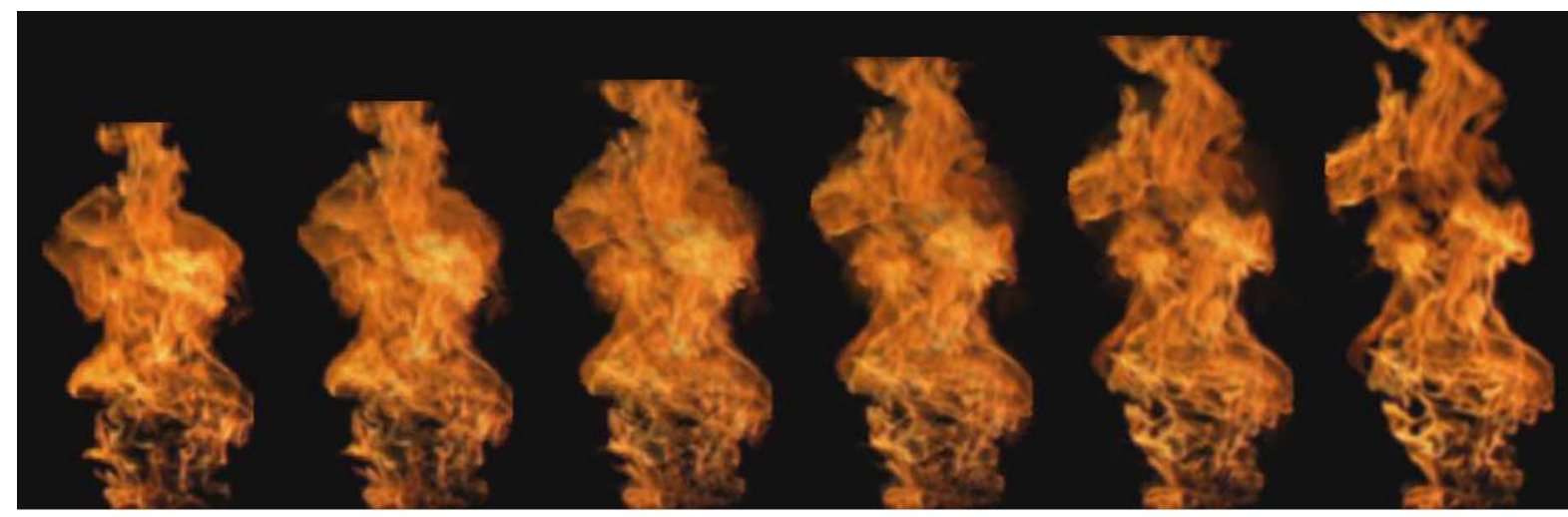

$(\mathrm{t}=0,0.2,0.4,0.6,0.8$ and 1$)$

Fig. 5. Morphing sequence of the flame imagery using MI as the comparison term.

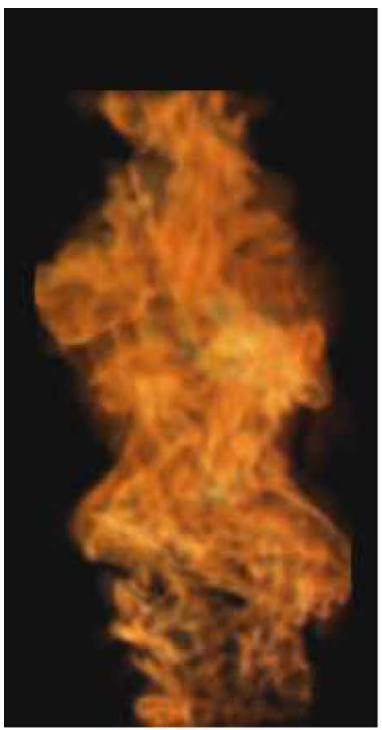

(a)

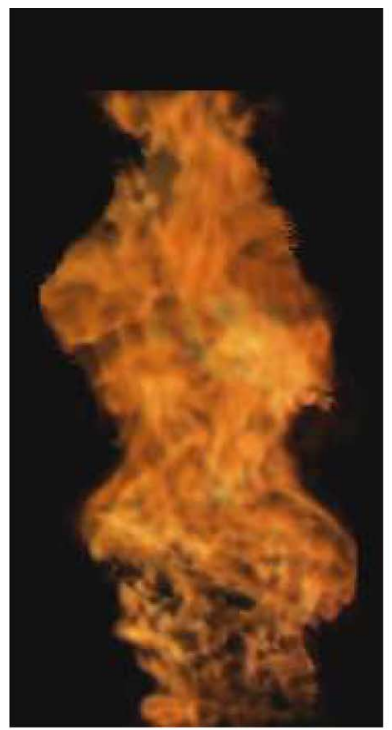

(b)

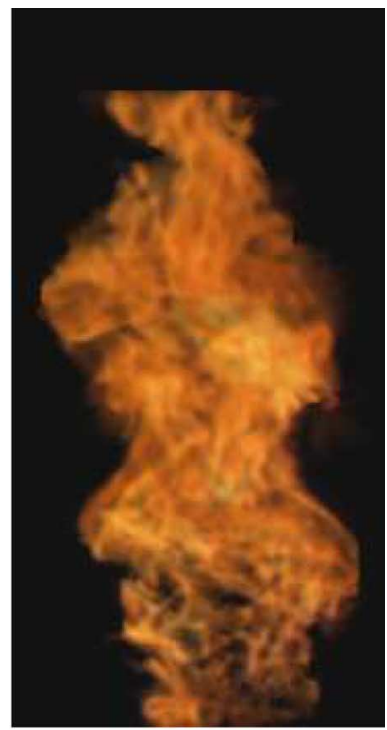

(c)

Fig. 6. Comparing results generated by pure $L^{2}$ MKP, SSD, and MI at $t=0.5$. (a) Pure $L^{2}$ MKP; (b) SSD; (c) MI.

leftmost column of Fig. 8 are the key frames selected from the original video sequence, and an image other then those in the leftmost column comes from the morphing result between the first image in its row and the first image in the next row. The last key frame is omitted and not shown here.

The third example illustrates image morphing over two doubly connected domains. Two $256 \times 256 \mathrm{MR}$ images of a human's heart were acquired using a GE MRI scanner at the diastolic [Fig. 9(a)] and the systolic [Fig. 9(b)] phases of the cardiac cycle. Dark regions in Fig. 10(a) and (b) are two doubly connected domains, corresponding to the myocardium and other tissues other than the heart ventricle. We also use image intensity as the mass density in this case, given the fact that MR image intensity is a function of photon density and, thus, related to tissue mass density. The dark regions in Fig. 10 are chosen as natural candidates to apply MP morphing to (in contrast to the heart ventricle region in which the change in mass is too drastic to satisfy the MP condition). Harmonic parameterization is first performed over each of the two doubly connected domains
(Fig. 11 shows the parameterization over the distolic phase image), and an FEM-based $L^{2}$ MKP is solved between the two domains to find the correspondence. In this example, pure $L^{2}$ MKP without comparison terms is adopted. Fig. 12 shows the final deformed grids. The in-between images can then be generated by cross dissolving over the entire domain, where the deformation inside the heart ventricle is obtained by 2-D spatial interpolation. Fig. 13 shows selected frames from the morphing result.

\section{CONCLUSIONS AND DISCUSSION}

In this paper, we applied a modified Monge-Kantorovich flow to the problem of image morphing by adding a comparison term to the optimal mass transport energy functional. Other than the morphing examples presented in this paper, this technique can also be used for the problem of medical image registration, if the underlying physics model satisfies the mass preserving condition. We are currently using the image intensity or a smoothed version of image intensity as the mass 


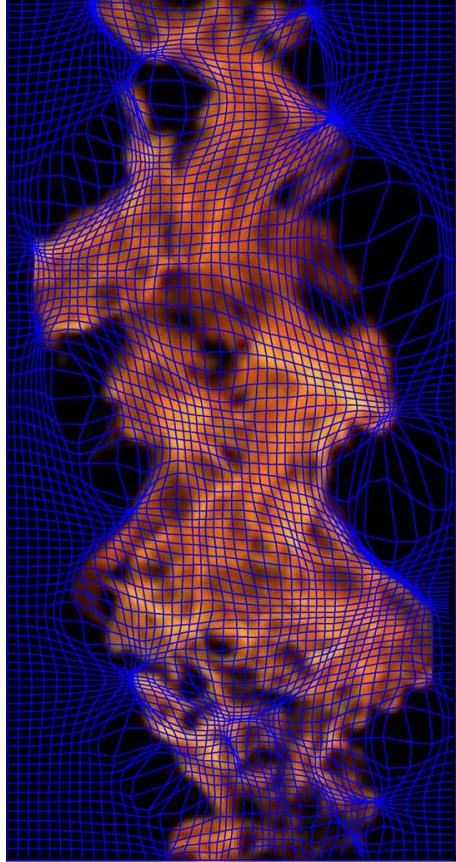

(a)

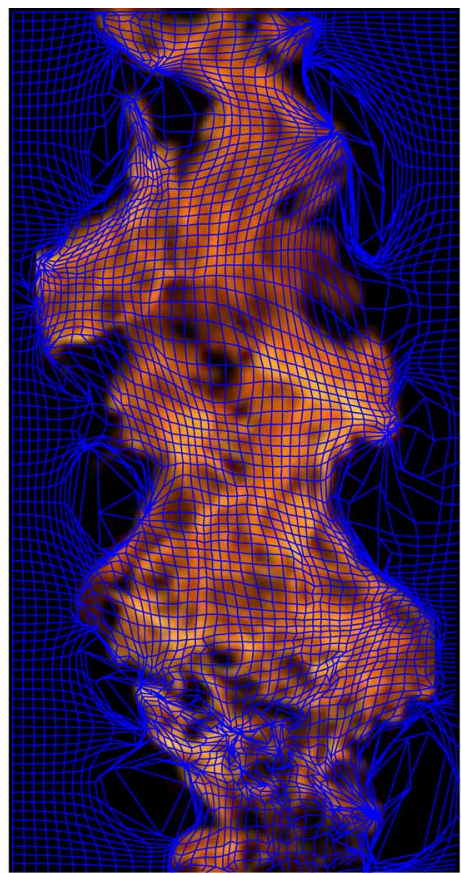

(b)

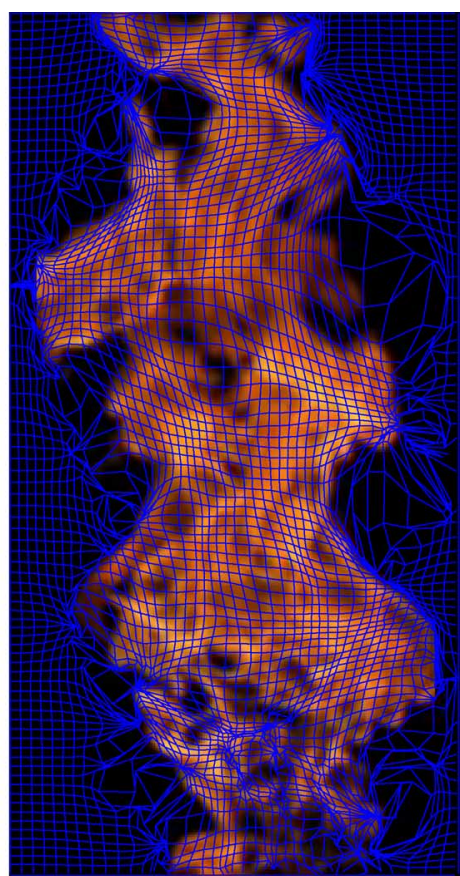

(c)

Fig. 7. Comparing deformed grids generated by pure $L^{2}$ MKP, SSD, and MI. (a) Pure $L^{2}$ MKP; (b) SSD; (c) MI.

density, which is the simplest mapping function from intensity to mass. This requires the initial and end image to be similar in the sense that they have the same amount of mass in order to satisfy the mass-preserving condition. For images with only certain regions that satisfy this condition, we perform image segmentation first and apply the morphing method only on the mass-preserving regions. This can be done through solving the $L^{2}$ MKP between two doubly connected domains. If the inner boundary is small enough, it can be regarded as a landmark, and, hence, we have solved the problem of MP morphing with a single landmark. This technique can be extended to multiconnected domains (corresponding to multiple landmarks), simply by dividing each domain into several doubly connected domains to build the initial mapping [38].

The $L^{2}$ mass moving penalty studied in this paper may be sometimes too severe. It tends to favor changes in density over moving the mass around. In fact, this is the main reason that causes the double exposure effects commonly seen in image morphing applications. $L^{1}$ type mass moving penalty is much less severe for large movement, but it may produce nonsmooth results. To solve this problem, we are considering the use of $L^{1+\epsilon}$ penalty in our future research, with $\epsilon$ being a value between 0 and 1 .

The MP constraint can also be combined with the concept of harmonic mapping to provide a new approach of MP diffeomorphisms, i.e. to consider the minimization of the Dirichlet integral over all MP maps

$$
\min _{u \in M P} \int_{\Omega_{0}}\|D u\|^{2} .
$$

The underlying physical assumption of MP mapping is exactly the same as that of extended optical flow constraint
(EOFC) [4]. Developing new optical flow algorithms within the framework of optimal mass preserving mapping can also be an interesting future direction.

\section{APPENDIX}

In this Appendix, we give mathematical details omitted in the main sections of this paper. We will mainly focus on the proof of MP mapping properties and the derivation of our improved gradient descent method.

\section{A. Properties of Mass Preserving (MP) Mappings}

We have stated in Section III-B that in order to preserve the MP condition, the evolution of $s$ and $u$ must satisfy

$$
\begin{aligned}
s_{t} & =\left(\frac{1}{\mu_{0}} \zeta\right) \circ s \\
u_{t} & =-\frac{1}{\mu_{0}} D u \zeta
\end{aligned}
$$

for some vector field $\zeta$ on $\Omega_{0}$, with $\operatorname{div}(\zeta)=0$ and $\langle\zeta, \vec{n}\rangle=0$ on $\partial \Omega_{0}, \vec{n}$ being the normal to the boundary of $\Omega_{0}$. Now we give the proof to this statement. Since $s$ is an MP mapping from $\left(\Omega_{0}, \mu_{0}\right)$ to itself, according to the Jacobian constraint we have $\mu_{0}=|D s| \mu_{0} \circ s$. By differentiating it with respect to time $t$, we get

$$
\begin{aligned}
& 0=|D s|_{t} \mu_{0} \circ s+|D s|\left(\mu_{0} \circ s\right)_{t} \\
& 0=|D s|\left(\operatorname{div}\left(s_{t} \circ s^{-1}\right) \circ s\right) \mu_{0} \circ s+|D s|\left\langle\left(\nabla \mu_{0}\right) \circ s, s_{t}\right\rangle \\
& 0=\left(\mu_{0} \operatorname{div}\left(s_{t} \circ s^{-1}\right)\right) \circ s+\left\langle\left(\nabla \mu_{0}\right) \circ s, s_{t}\right\rangle \\
& 0=\mu_{0} \operatorname{div}\left(s_{t} \circ s^{-1}\right)+\left\langle\nabla \mu_{0}, s_{t} \circ s^{-1}\right\rangle \\
& 0=\operatorname{div}\left(\mu_{0} s_{t} \circ s^{-1}\right) .
\end{aligned}
$$



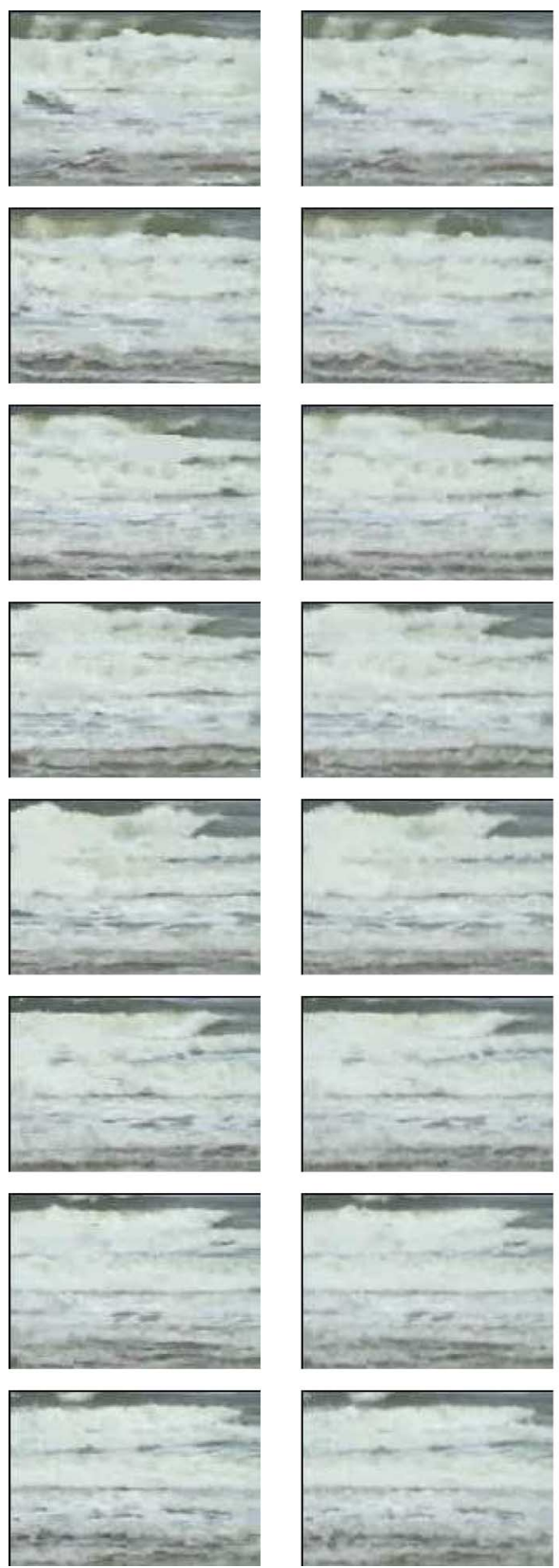
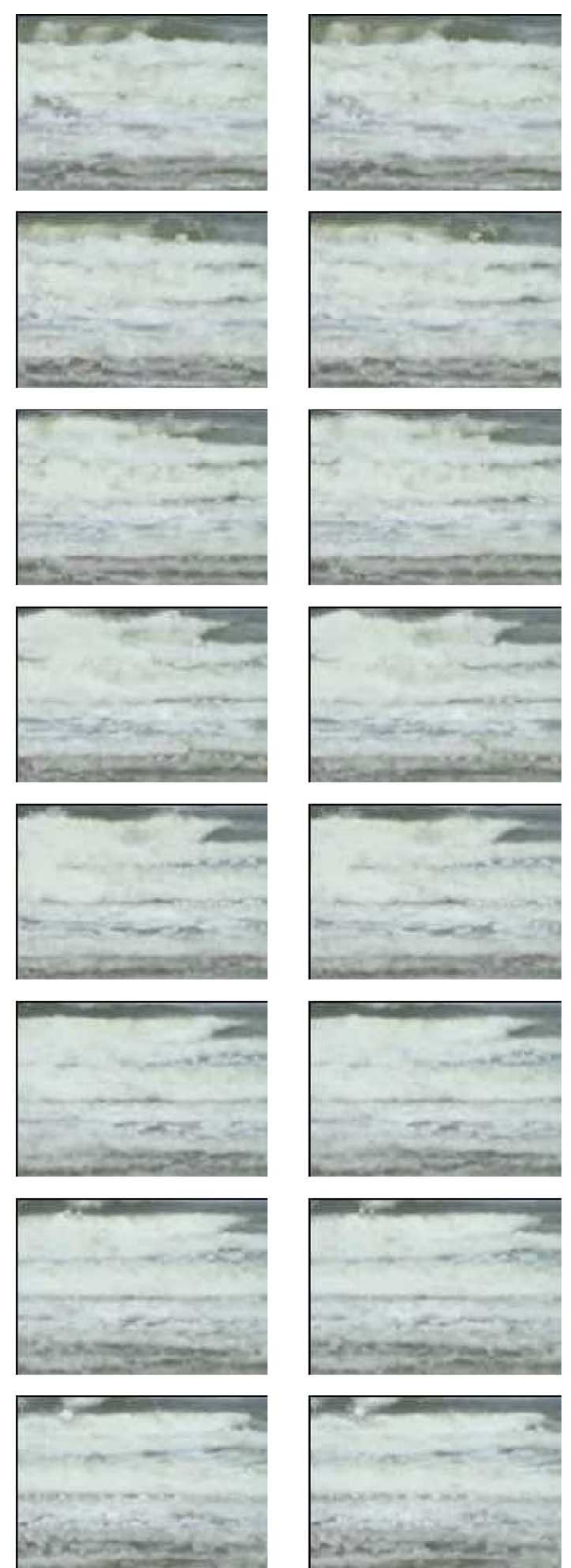
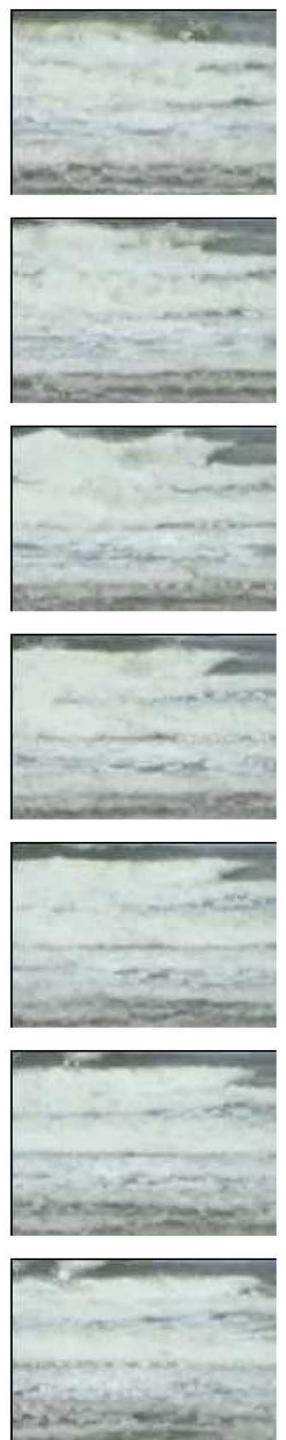
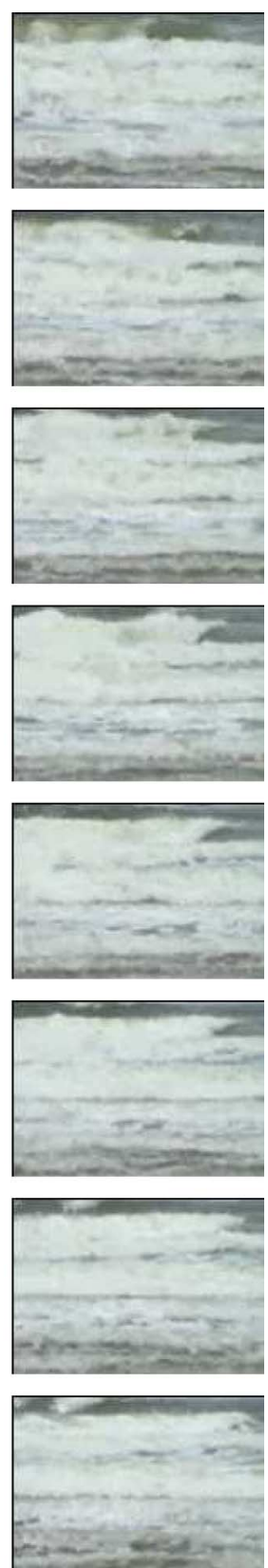

Fig. 8. Morphing sequence of a wave imagery. Leftmost column: selected key frames. Others: Interpolated images. SSD used as the comparison term.

Hence, $s_{t}$ must have the following form:

$$
s_{t}=\left(\frac{1}{\mu_{0}} \zeta\right) \circ s
$$

for some vector field $\zeta$ on $\Omega_{0}$, with $\operatorname{div}(\zeta)=0$ and $\langle\zeta, \vec{n}\rangle=0$ on $\partial \Omega_{0}, \vec{n}$ being the normal to the boundary of $\Omega_{0}$. Since $u$ and $s$ satisfy

$$
u \circ s=u^{0}
$$

by taking the derivative of (36) with respect to $t$, we have

$$
\begin{aligned}
(D u \circ s) s_{t}+u_{t} \circ s & =0, \\
u_{t} \circ s & =-(D u \circ s) s_{t} \\
u_{t} & =-D u s_{t} \circ s^{-1} .
\end{aligned}
$$

From (35), we get

$$
u_{t}=-\frac{1}{\mu_{0}} D u \zeta
$$

\section{B. Gradient Descent Methods for Pure $L^{2} M K P$}

In the following discussion, we intend to minimize a more general form of the energy functional

$$
M[u]=\int_{\Omega_{0}} \Phi(u(x)-x) \mu_{0}(x) d x
$$

where $\Phi$ is a non-negative $C^{1}$ function. When $\Phi(x)=\|x\|^{2}$, it is exactly the $L^{2}$ Kantorovich-Wasserstein functional. The first step is to find an initial MP mapping $u^{0}$ as stated in Section III-A. The second step is to minimize the energy functional 


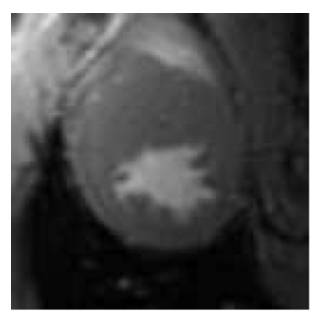

(a)

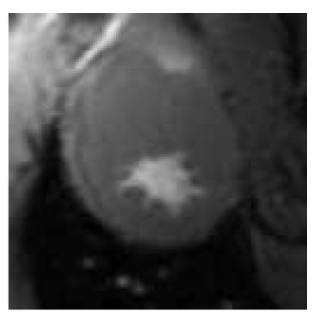

(b)
Fig. 9. Two MR heart images. (a) Diastolic phase; (b) systolic phase.

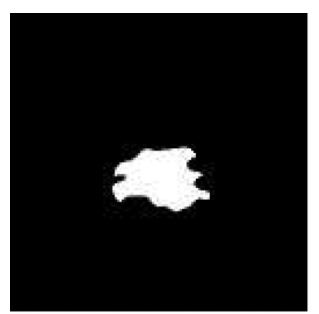

(a)

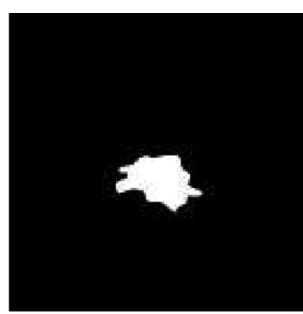

(b)
Fig. 10. Segmentation results of the heart images in Fig. 9. (a) Diastolic phase; (b) systolic phase.

over $u=u^{0} \circ s^{-1}$ by varying $s$ over MP mappings from $\Omega_{0}$ to $\Omega_{0}$, starting with $s$ equal to the identity map.

A change of variable is applied here by substituting $x$ in (38) with $y=s^{-1}(x)$. Due to the MP property of $s$ and $s^{-1}$, the following is obvious:

$\mu_{0}(x) d x=\mu_{0} \circ s(y) d s(y)=\mu_{0} \circ s(y)|D s(y)| d y=\mu_{0}(y) d y$

and also $u(x)=u \circ s(y)=u^{0}(y)$. Hence, functional (38) equals

$$
M=\int_{\Omega_{0}} \Phi\left(u^{0}(y)-s(y)\right) \mu_{0}(y) d y .
$$

By taking the derivative of (40) with respect to $t$, we get

$$
\frac{d M}{d t}=-\int_{\Omega_{0}}\left\langle\nabla \Phi\left(u^{0}(y)-s(y)\right), \frac{\partial s}{\partial t}(y)\right\rangle \mu_{0}(y) d y \text {. }
$$

Then we do another change of variable by substituting $y$ back with $x=s(y)$ and get

$$
\frac{d M}{d t}=-\int_{\Omega_{0}}\left\langle\nabla \Phi(u(x)-x), \mu_{0}(x) s_{t} \circ s^{-1}(x)\right\rangle d x .
$$

Clearly, were it not for the constraint $\operatorname{div}\left(\mu_{0} s_{t} \circ s^{-1}\right)=0$, we could take $\mu_{0} s_{t} \circ s^{-1}=\nabla \Phi(u(x)-x)$ to decrease energy. However, considering this MP constraint, $\mu_{0} s_{t} \circ s^{-1}$ should be a divergence-free vector field. Hence, we define

$$
\zeta=\mu_{0}\left(s_{t} \circ s\right)^{-1}
$$

$\nabla \Phi(u(x)-x)$ can be decomposed into a curl-free part and a divergence-free part (Helmholtz decomposition [29]), i.e.,

$$
\nabla \Phi(u(x)-x)=\nabla w+\chi
$$

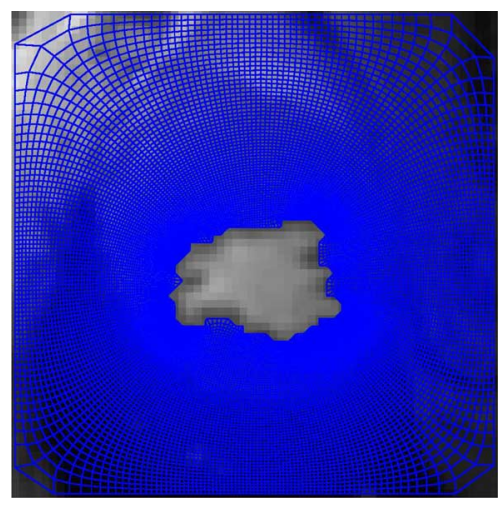

Fig. 11. Harmonic parameterization of the heart image in the diastolic phase.

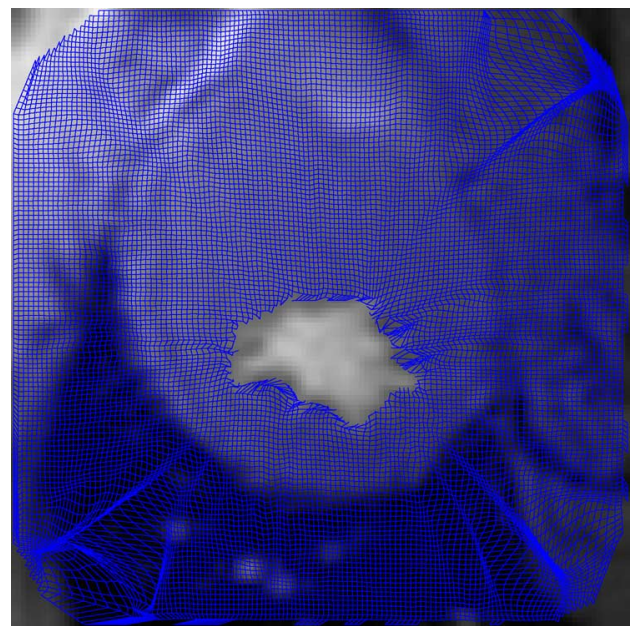

Fig. 12. Deformed grids over the heart image in the systolic phase.

where $\operatorname{div}(\chi)=0$ and $\langle\chi, \vec{n}\rangle=0$ on $\partial \Omega_{0}$. Then, (42) can be rewritten as

$$
\begin{aligned}
-M_{t} & =\int_{\Omega_{0}}\langle\nabla w+\chi, \zeta\rangle \\
& =\int_{\Omega_{0}}\langle\nabla w, \zeta\rangle+\int_{\Omega_{0}}\langle\chi, \zeta\rangle \\
& =\int_{\Omega_{0}}(\operatorname{div}(w \zeta)-w \operatorname{div}(\zeta))+\int_{\Omega_{0}}\langle\chi, \zeta\rangle \\
& =\int_{\partial \Omega_{0}} w\langle\zeta, n\rangle+\int_{\Omega_{0}}\langle\chi, \zeta\rangle=\int_{\Omega_{0}}\langle\chi, \zeta\rangle
\end{aligned}
$$

where $\zeta$ is chosen to be $\chi$, and can be found through the Helmholtz decomposition.

Now we give the evolving equation for $u$ in the general $\mathbf{R}^{\mathbf{d}}$ case, as well as in the 2-D case which has a simpler expression and will be used in our algorithm.

1) Gradient Descent in $\mathbf{R}^{d}$ : By taking the divergence of (44) on both sides, it is easy to see that $w$ is a solution of the following Neumann-type boundary problem

$$
\begin{aligned}
\operatorname{div}(\nabla \Phi(u(x)-x)) & =\Delta w \\
\langle\nabla w, \vec{n}\rangle & =\langle\nabla \Phi(u(x)-x), \vec{n}\rangle \text { on } \partial \Omega_{0}
\end{aligned}
$$



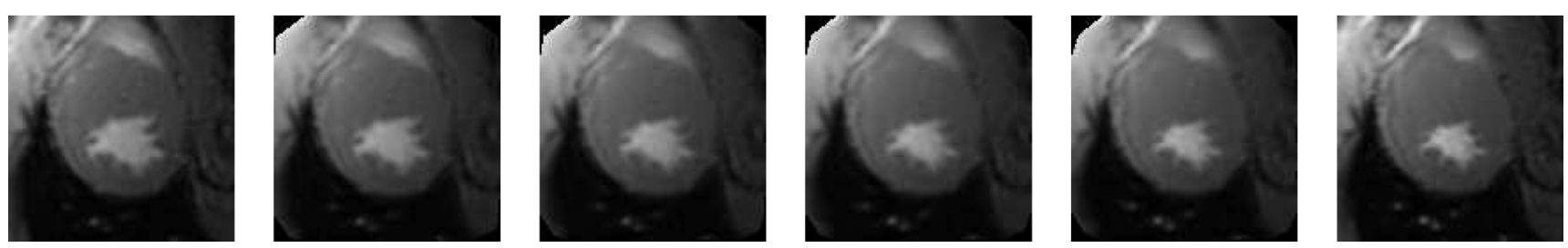

Fig. 13. Morphing sequence of the heart imagery using two given images in Fig. 9.

and we can set $\chi=\nabla \Phi(u(x)-x)-\nabla w$. It is then easily seen that $\chi$ satisfies the MP constraints. This PDE can be solved using a number of available methods, such as the finite-volume method. Thus, by (37), we have the following evolution equation for $u$ :

$$
u_{t}=-\frac{1}{\mu_{0}} D u\left\{\nabla \Phi(u(x)-x)-\nabla \Delta^{-1} \operatorname{div}[\nabla \Phi(u(x)-x)]\right\}
$$

which is a first-order nonlocal scheme for $u_{t}$, if we count $\Delta^{-1}$ as minus 2 derivatives.

If $\Phi(u(x)-x)$ has the form of $\|u(x)-x\|^{2}$, which is the $L^{2}$ MKP, (47) has the form of

$$
u_{t}=-\frac{2}{\mu_{0}} D u\left\{u(x)-x-\nabla \Delta^{-1} \operatorname{div}[u(x)-x]\right\} .
$$

2) Gradient Descent in $\mathbf{R}^{2}$ : The situation is simpler in the $\mathbf{R}^{2}$ case, due to the fact that a divergence free vector field $\zeta$ can be written as $\zeta=\nabla^{\perp} h$ for a scalar function $h$, where $\perp$ represents rotation by $\pi / 2$ counter clockwise, so that $\nabla^{\perp} h=$ $\left(-h_{y}, h_{x}\right)$. In this case, (45) becomes

$$
-M_{t}=\int_{\Omega_{0}}\left\langle\nabla^{\perp} f, \nabla^{\perp} h\right\rangle=\int_{\Omega_{0}}\langle\nabla f, \nabla h\rangle
$$

where the decomposition of $\nabla \Phi(u(x)-x)$ is $\nabla \Phi(u(x)-x)=$ $\nabla w+\nabla^{\perp} f$, and we can let $h$ equal $f$. Hence

$$
\nabla \Phi(u(x)-x)^{\perp}=\nabla^{\perp} w-\nabla f .
$$

Considering

$$
\operatorname{div}\left(\nabla^{\perp} w\right)=\operatorname{div}\left(-w_{y}, w_{x}\right)=-w_{y x}+w_{x y}=0
$$

function $f$ can be solved by the following Dirichlet-type boundary problem

$$
\begin{aligned}
-\operatorname{div}\left(\nabla \Phi(u(x)-x)^{\perp}\right) & =\Delta f \\
f & =0 \text { on } \partial \Omega_{0}
\end{aligned}
$$

which gives us the evolution equation

$$
u_{t}=\frac{1}{\mu_{0}} D u \nabla^{\perp} \Delta^{-1} \operatorname{div}\left(\nabla \Phi(u(x)-x)^{\perp}\right) .
$$

In the $L^{2} \mathrm{MKP},(53)$ can be rewritten as

$$
u_{t}=\frac{2}{\mu_{0}} D u \nabla^{\perp} \Delta^{-1} \operatorname{div}\left((u-\underline{i d})^{\perp}\right)
$$

where $\underline{i d}$ is an identity map.

\section{Gradient Descent Methods for Energy Functionals With a Comparison Term}

We now rewrite the energy functional as the sum of two terms

$$
M=M_{1}+\alpha M_{2}
$$

with $M_{1}$ being the comparison term penalizing the change in intensity. The second term of the energy functional $M_{2}=\int\|u(x)-x\|^{2} \mu_{0} d x$ is exactly the $L^{2} \mathrm{MKP}$, which has been discussed above. We focus on the first term $M_{1}$, and discuss the cases of SSD and MI, respectively.

1) SSD as the Comparison Term: If SSD is employed as the comparison, then

$$
M_{1}=\int\left(I_{1} \circ u-I_{0}\right)^{2} d x .
$$

Before taking the derivative, we multiply $M_{1}$ by $\mu_{0}$ and divide by $\mu_{0}$, i.e.,

$$
M_{1}=\int\left[\frac{1}{\mu_{0}}\left(I_{1} \circ u-I_{0}\right)^{2}\right] \mu_{0} d x \text {. }
$$

By setting $y=s^{-1}(x)$ and considering (39): $\mu_{0}(x) d x=$ $\mu_{0}(y) d y$, we get

$$
\begin{aligned}
& M_{1}=\int\left[I_{1} \circ u(x)-I_{0}(x)\right]^{2} d x \\
& M_{1}=\int \frac{1}{\mu_{0}(x)}\left[I_{1} \circ u(x)-I_{0}(x)\right]^{2} \mu_{0}(x) d x \\
& M_{1}=\int \frac{1}{\mu_{0} \circ s(y)}\left[I_{1} \circ u^{0}(y)-I_{0} \circ s(y)\right]^{2} \mu_{0}(y) d y .
\end{aligned}
$$

Then we take the derivative of $M_{1}$ with respect to $t$ and find that

$$
\begin{aligned}
M_{1 t}=\int\langle & -\frac{1}{\mu_{0}^{2} \circ s(y)}\left[I_{1} \circ u^{0}(y)-I_{0} \circ s(y)\right]^{2} \\
& \times \nabla \mu_{0}(y) \circ s(y)-\frac{2}{\mu_{0} \circ s(y)} \\
& \left.\times\left[I_{1} \circ u^{0}(y)-I_{0} \circ s(y)\right] \nabla I_{0} \circ s(y), \frac{\partial s^{t}}{\partial t}\right\rangle \\
& \times \mu_{0}(y) d y .
\end{aligned}
$$

By changing $y$ back to $x$, we see that

$$
\begin{aligned}
& -M_{1 t}=\int\left\langle\frac{1}{\mu_{0}^{2}(x)}\left[I_{1} \circ u(x)-I_{0}(x)\right]^{2} \nabla \mu_{0}(x)\right. \\
& \left.+\frac{2}{\mu_{0}(x)}\left[I_{1} \circ u(x)-I_{0}(x)\right] \nabla I_{0}(x), \mu_{0} \frac{\partial s}{\partial t} \circ s^{-1}(x)\right\rangle d x .
\end{aligned}
$$


Adding the derivative of the $M_{2}$ term, $P$ is defined as

$P_{\mathrm{SSD}}=\frac{1}{\mu_{0}^{2}}\left(I_{1} \circ u-I_{0}\right)^{2} \nabla \mu_{0}+\frac{2}{\mu_{0}}\left(I_{1} \circ u-I_{0}\right) \nabla I_{0}+2 \alpha(u-\underline{i d})$

which is (19) in Section III.

2) MI as the Comparison Term: If MI is used as the comparison term

$$
M_{1}=-\int_{i_{0} \times i_{1}} p_{u}^{I_{0}, I_{1} \circ u}\left(i_{0}, i_{1}\right) \log \frac{p_{u}^{I_{0}, I_{1} \circ u}\left(i_{0}, i_{1}\right)}{p^{I_{0}}\left(i_{0}\right) p_{u}^{I_{1} \circ u}\left(i_{1}\right)} d i_{0} d i_{1} .
$$

Now we take the derivative of (61) respect to $t$ as in [16]

$$
\begin{aligned}
\frac{\partial M_{1}}{\partial t}= & -\frac{\partial}{\partial t} \int_{i_{0} \times i_{1}} p_{u}^{I_{0}, I_{1} \circ u}\left(i_{0}, i_{1}\right) \log \frac{p_{u}^{I_{0}, I_{1} \circ u}\left(i_{0}, i_{1}\right)}{p^{I_{0}}\left(i_{0}\right) p_{u}^{I_{1} \circ u}\left(i_{1}\right)} d i_{0} d i_{1} \\
= & -\int_{i_{0} \times i_{1}} \log \frac{p_{u}^{I_{0}, I_{1} \circ u}\left(i_{0}, i_{1}\right)}{p^{I_{0}}\left(i_{0}\right) p_{u}^{I_{1} \circ u}\left(i_{1}\right)} \frac{\partial p_{u}^{I_{0}, I_{1} \circ u}\left(i_{0}, i_{1}\right)}{\partial t} d i_{0} d i_{1} \\
& -\int_{i_{0} \times i_{1}} \frac{\partial p_{u}^{I_{0}, I_{1} \circ u}\left(i_{0}, i_{1}\right)}{\partial t} d i_{0} d i_{1} \\
& +\int_{i_{0} \times i_{1}} \frac{p_{u}^{I_{0}, I_{1} \circ u}\left(i_{0}, i_{1}\right)}{p_{u}^{I_{1} \circ u\left(i_{1}\right)}} \frac{\partial p_{u}^{I_{1} \circ u\left(i_{1}\right)}}{\partial t} d i_{0} d i_{1} .
\end{aligned}
$$

Considering

$$
\int_{i_{0}} p_{u}^{I_{0}, I_{1} \circ u}\left(i_{0}, i_{1}\right) d i_{0}=p_{u}^{I_{1} \circ u}\left(i_{1}\right)
$$

it is easy to see that the last term equals zero; hence, (62) can be written as the following:

$$
\begin{array}{r}
\frac{\partial M_{1}}{\partial t}=-\int_{i_{0} \times i_{1}}\left[1+\log \frac{p_{u}^{I_{0}, I_{1} \circ u}\left(i_{0}, i_{1}\right)}{p^{I_{0}}\left(i_{0}\right) p_{u}^{I_{1} \circ u}\left(i_{1}\right)}\right] \\
\times \frac{\partial p_{u}^{I_{0}, I_{1} \circ u}\left(i_{0}, i_{1}\right)}{\partial t} d i_{0} d i_{1} .
\end{array}
$$

where $p^{I_{0}}, p_{u}^{I_{1} \circ u}$, and $p_{u}^{I_{0}, I_{1} \circ u}$ can be computed using (7), (8), and (9), respectively. $(\partial / \partial t) p_{u}^{I_{0}, I_{1} \circ u}\left(i_{0}, i_{1}\right)$ can be computed as follows. First, (9) can be rewritten as

$$
\begin{aligned}
p_{u}^{I_{0}, I_{1} \circ u}\left(i_{0}, i_{1}\right)= & \frac{1}{V} \int_{\Omega_{0}} \frac{1}{\mu_{0}(x)} \psi \\
& \times\left(I_{0}(x)-i_{0}, I_{1}\left(u(x)-i_{1}\right)\right) \mu_{0}(x) d x
\end{aligned}
$$

where the same method of multiplying and dividing $\mu_{0}$ has been applied. Then by setting $y=s^{-1}(x)\left(s^{-1}\right.$ is an MP mapping from $\Omega_{0}$ onto itself) and noticing $u(x)=u^{0} \circ s^{-1}$, we get

$$
\begin{aligned}
p_{u}^{I_{0}, I_{1} \circ u}\left(i_{0}, i_{1}\right) & =\frac{1}{V} \int_{\Omega_{0}} \frac{1}{\mu_{0} \circ s(y)} \psi \\
& \left.\times\left(I_{0}(s(y))-i_{0}, I_{1}\left(u^{0}(y)\right)-i_{1}\right)\right) \mu_{0}(y) d y .
\end{aligned}
$$

The derivative of $p_{u}^{I_{0}, I_{1} \circ u}\left(i_{0}, i_{1}\right)$ is given as

$$
\begin{aligned}
& \frac{\partial p_{u}^{I_{0}, I_{1} \circ u}}{\partial t}\left(i_{0}, i_{1}\right) \\
& =\frac{1}{V} \int_{\Omega_{0}}\left\langle\frac{\psi_{\alpha}\left(I_{0}(s(y))-i_{0}, I_{1}\left(u^{0}(y)\right)-i_{1}\right)}{\mu_{0} \circ s(y)} \nabla I_{0}(s(y))\right. \\
& \quad-\frac{\psi\left(I_{0}(s(y))-i_{0}, I_{1}\left(u^{0}(y)\right)-i_{1}\right)}{\mu_{0}^{2} \circ s(y)} \nabla \mu_{0}(s(y)) \\
& \left.\quad \frac{\partial s(y)}{\partial t}\right\rangle \mu_{0}(y) d y
\end{aligned}
$$

where $\psi_{\alpha}$ is the partial derivative of $\psi$ with respect to its first component. Now, we do the change of variable again by substituting $y$ with $s^{-1}(x)$ and derive

$$
\begin{gathered}
\frac{d p_{u}^{I_{0}, I_{1} \circ u}}{d t}\left(i_{0}, i_{1}\right) \\
=\frac{1}{V} \int\left\langle\frac{\psi_{\alpha}\left(I_{0}(x)-i_{0}, I_{1}(u(x))-i_{1}\right)}{\mu_{0}(x)} \nabla I_{0}(x)\right. \\
-\frac{\psi\left(I_{0}(x)-i_{0}, I_{1}(u(x))-i_{1}\right)}{\mu_{0}^{2}(x)} \nabla \mu_{0}(x), \\
\left.\frac{\partial s}{\partial t} \circ s^{-1}(x)\right\rangle \mu_{0}(x) d x .
\end{gathered}
$$

Hence, the derivative of $M_{1}$ with respect to $t$ is given by (69), shown at the bottom of the page. Equation (69) is a quadruple integral, which could be rewritten in a concise form by using convolution

$$
\begin{aligned}
\frac{\partial M_{1}}{\partial t}=\int_{\Omega_{0}}\left\langle-\frac{1}{V}[\right. & \left(1+\log \frac{p_{u}^{I_{0}, I_{1} \circ u}}{p^{I_{0}} p^{I_{1} \circ u}}\right) * \psi_{\alpha}\left(I_{0}(x), I_{1} \circ u(x)\right) \\
& \times \frac{\nabla I_{0}(x)}{\mu_{0}(x)}-\left(1+\log \frac{p_{u}^{I_{0}, I_{1} \circ u}}{p^{I_{0}} p^{I_{1} \circ u}}\right) \\
& \left.* \psi\left(I_{0}(x), I_{1} \circ u(x)\right) \frac{\nabla \mu_{0}(x)}{\mu_{0}^{2}(x)}\right] \\
\frac{\partial s}{\partial t} & \left.\circ s^{-1}(x)\right\rangle \mu_{0} d x .
\end{aligned}
$$

$$
\begin{array}{r}
\frac{\partial M_{1}}{\partial t}=-\int_{i_{0} \times i_{1}} \int_{\Omega_{0}}\left\langle\frac { 1 } { V } [ 1 + \operatorname { l o g } \frac { p _ { u } ^ { I _ { 0 } , I _ { 1 } \circ u } ( i _ { 0 } , i _ { 1 } ) } { p ^ { I _ { 0 } } ( i _ { 0 } ) p _ { u } ^ { I _ { 1 } \circ u } ( i _ { 1 } ) } ] \left[\frac{\psi_{\alpha}\left(I_{0}(x)-i_{0}, I_{1}(u(x))-i_{1}\right)}{\mu_{0}(x)} \nabla I_{0}(x)\right.\right. \\
\left.\left.-\frac{\psi\left(I_{0}(x)-i_{0}, I_{1}(u(x))-i_{1}\right)}{\mu_{0}^{2}(x)} \nabla \mu_{0}(x)\right], \frac{\partial s}{\partial t} \circ s^{-1}(x)\right\rangle \mu_{0}(x) d x d i_{0} d i_{1}
\end{array}
$$


Now it has the form of a double integral on $\Omega_{0}$ domain, and can be combined with the derivative from the $M_{2}$ term. Thus, $P$ is

$$
\begin{aligned}
P_{M I}=-\frac{1}{V}[ & \left(1+\log \frac{p_{u}^{I_{0}, I_{1} \circ u}}{p^{I_{0}} p^{I_{1} \circ u}}\right) * \psi_{\alpha}\left(I_{0}(x), I_{1} \circ u(x)\right) \\
& \times \frac{\nabla I_{0}(x)}{\mu_{0}(x)}-\left(1+\log \frac{p_{u}^{I_{0}, I_{1} \circ u}}{p^{I_{0}} p^{I_{1} \circ u}}\right) \\
& \left.* \psi\left(I_{0}(x), I_{1} \circ u(x)\right) \frac{\nabla \mu_{0}(x)}{\mu_{0}^{2}(x)}\right] \\
+ & 2 \alpha(u-\underline{i d})
\end{aligned}
$$

which is (20).

It is easy to deduce the evolution equation for $u$, using the same approach for pure $L^{2}$ MKP. In the $\mathbf{R}^{\mathbf{d}}$ case, we find a scalar function $w$ to be the solution of the following Neumanntype boundary problem

$$
\begin{aligned}
\operatorname{div}(P) & =\Delta w \\
\langle\nabla w, \vec{n}\rangle & =\langle P, \vec{n}\rangle \text { on } \partial \Omega_{0}
\end{aligned}
$$

and we can set $\chi=P-\nabla w$. The evolution equation for $u$ is given by

$$
u_{t}=-\frac{1}{\mu_{0}} D u\left\{P-\nabla \Delta^{-1} \operatorname{div}[\nabla \Phi(u(x)-x)]\right\}
$$

with $P$ being either $P_{\mathrm{SSD}}$ or $P_{M I}$.

In the $\mathbf{R}^{\mathbf{2}}$ case, function $f$ can be solved by the following Dirichlet-type boundary problem

$$
\begin{aligned}
-\operatorname{div}\left(P^{\perp}\right) & =\Delta f \\
f & =0 \text { on } \partial \Omega_{0}
\end{aligned}
$$

and the evolution equation for $u$ is given by

$$
u_{t}=\frac{1}{\mu_{0}} D u \nabla^{\perp} \Delta^{-1} \operatorname{div}\left(P^{\perp}\right) .
$$

\section{REFERENCES}

[1] S. Angenent, S. Haker, and A. Tannenbaum, "Minimizing flows for the Monge-Kantorovich problem," SIAM J. Math. Anal., vol. 35, no. 1, pp. 61-97, 2003.

[2] T. Beier and S. Neely, "Feature-based image metamorphosis," in Proc. SIGGRAPH, 1992, vol. 26, pp. 35-42.

[3] J. D. Benamou and Y. Brenier, "A computational fluid mechanics solution to the Monge-Kantorovich mass transfer problem," Numer. Math., vol. 84, pp. 375-393, 2000.

[4] A. Bimbo, "Optical flow computation using extended constraints," IEEE Trans. Image Process., vol. 5, no. 5, pp. 720-739, May 1996.

[5] Y. Brenier, "Polar factorization and monotone rearrangement of vectorvalued functions," Commun. Pure Appl. Math., vol. 64, pp. 375-417, 1991.

[6] G. E. Christensen, R. D. Babbitt, and M. I. Miller, "Deformable templates using large deformation kinematics," IEEE Trans. Image Process., vol. 5, no. 10, pp. 1435-1447, Oct. 1996.

[7] M. Cullen and R. Purser, "An extended lagrangian theory of semigeostrophic frontogenesis," J. Atmos. Sci., vol. 41, pp. 1477-1497, 1984.

[8] B. Dacorogna and J. Moser, "On a partial differential equation involving the Jacobian determinant," Ann. Inst. H. Poincaré Anal. Non Linéaire, vol. 7, pp. 1-26, 1990.

[9] R. Duda, P. Hart, and D. Stork, Pattern Classification. : Wiley-Interscience, 2000.

[10] R. Fattal and D. Lischinski, "Target-driven smoke animation," $A C M$ Trans. Graph., vol. 23, no. 3, pp. 441-448, 2004.
[11] W. Gangbo and R. McCann, "The geometry of optimal transportation," Acta Math., vol. 177, pp. 113-161, 1996.

[12] E. Haber and J. Mondersitzki, "Numerical methods for volume preserving image registration," Inv. Probl., vol. 20, pp. 1621-1638, 2004.

[13] S. Haker, S. Angenent, A. Tannenbaum, and R. Kikinis, "Nondistorting flattening maps and the 3D visualization of colon CT images," IEEE Trans. Med. Imag., vol. 19, no. 7, pp. 665-670, Jul. 2000.

[14] S. Haker, L. Zhu, A. Tannenbaum, and S. Angenent, "Optimal mass transport for registration and warping," Int. J. Comput. Vis., vol. 60, no. 3, pp. 225-240, 2004.

[15] A. Hassanien and M. Nakajima, "Image morphing of facial images transformation based on navier elastic body splines," in Proc. IEEE Computer Animation, 1998, vol. 8, pp. 119-125.

[16] G. Hermosillo, C. Chefd'Hotel, and O. Faugeras, "A variational approach to multi-modal image matching," Int. J. Comput. Vis., vol. 50, no. 3, pp. 329-343, 2002.

[17] M. Iwanowski, "Image morphing based on morphological interpolation combined with linear filtering,," J. WSCG, 2002.

[18] T. Kaijser, "Computing the Kantorovich distance for images," J. Math Imag. Vis., vol. 9, pp. 173-191, 1998.

[19] L. Kantorovich, "On a problem of Monge," Uspekhi Mat. Nauk., vol. 3, pp. 225-226, 1948.

[20] M. Knott and C. Smith, "On the optimal mapping of distributions," $J$. Optim. Theory, vol. 43, pp. 39-49, 1984.

[21] S.-Y. Lee, K.-Y. Chwa, J. Hahn, and S. Y. Shin, "Image morphing using deformation techniques," J. Vis. Comput. Anim., vol. 7, no. 1, pp. 3-23, 1996.

[22] E. Levina and P. Bickel, "The earth mover's distance is the mallow's distance: Some insights from statistics," in Proc. IEEE Int. Conf. Computer Vision, 2001, pp. 251-256.

[23] R. McCann, "Polar factorization of maps on riemannian manifolds," Geom. Funct. Anal., vol. 11, pp. 589-608, 2001.

[24] A. McNamara, A. Treuille, Z. Popovic, and J. Stam, "Fluid control using the adjoint method," ACM Trans. Graph., vol. 23, no. 3, pp. 449-456, 2004.

[25] M. I. Miller and L. Younes, "Group actions, homeomorphisms, and matching: A general framework," Int. J. Comput. Vis., vol. 41, no. 1-2, pp. 61-84, 2001.

[26] S. Rachev and L. Ruschendorf, Mass Transportation Problems. New York: Springer, 1998, vol. I \& II.

[27] Y. Rubner, "Perceptual metrics for image database navigation," Ph.D. dissertation, Stanford Univ., Stanford, CA, , May 1999.

[28] Y. Rubner, C. Tomasi, and J. Guibas, "The Earth Mover's Distance as a Metric for Image Retrieval," Tech. Rep. STAN-CS-TN-98-86 Dept. Comput. Sci., Stanford Univ., Stanford, CA, Sep. 1998.

[29] G. Strang, Introduction to Applied Mathematics. Wellesley, MA: Ellesley-Cambridge, 1986.

[30] H. Tagare, "Deformable 2-d template matching using orthogonal curves," IEEE Trans. Med. Imag., vol. 16, no. 1, pp. 108-117, Jan. 1997.

[31] A. Toga, Brain Warping. San Diego, CA: Academic, 1999.

[32] A. Trouve, "Diffeomorphism groups and pattern matching in image analysis," Int. J. Comput. Vis., vol. 28, no. 3, pp. 213-221, 1998.

[33] A. Treuille, A. McNamara, Z. Popovic, and J. Stam, "Keyframe control of smoke simulations," ACM Trans. Graph., vol. 22, no. 3, pp. 716-723, 2003.

[34] P. Viola and W. M. Wells, III, "Alignment by maximization of mutual information," Int. J. Comput. Vis., vol. 24, no. 2, pp. 137-154, 1997.

[35] G. Wolberg, "Recent advances in image morphing," in Proc. Int. Conf. Comput. Graph., 1996, pp. 64-71, IEEE.

[36] A. Yezzi and J. Prince, "An eulerian PDE approach for computing tissue thickness," IEEE Trans. Med. Imag., vol. 22, no. 10, pp. 1332-1339, Oct. 2003.

[37] C. Zhang and F. S. Cohen, "3-D face structure extraction and recognition from images using 3-D morphing and distance mapping," IEEE Trans. Image Process., vol. 11, no. 11, pp. 1249-1259, Nov. 2002.

[38] L. Zhu, "On visualizing branched surface: An angle/area preserving approach," Ph.D. dissertation, Georgia Inst. Technol., Atlanta, 2004.

[39] L. Zhu, S. Haker, and A. Tannenbaum, "Flattening maps for the visualization of multibranched vessels," IEEE Trans. Med. Imag., vol. 24, no. 2, pp. 191-198, Feb. 2005.

[40] L. Zhu, Y. Yang, A. Tannenbaum, and S. Haker, "Image morphing based on mutual information and optimal mass transport," in Proc. Int. Conf. Image Process., 2004, pp. 1675-1678.

[41] L. Zhu, S. Haker, and A. Tannenbaum, "Mass preserving registration for heart MR images," in Proc. MICCAI, pp. 147-154. 


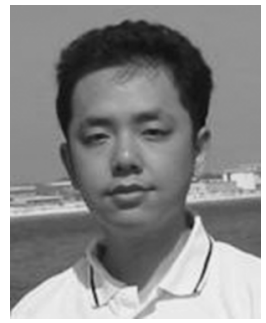

Lei Zhu received the B.S. and M.S. degrees in biomedical engineering from Tsinghua University, Beijing, China, in 1998 and 2000, respectively, and the Ph.D. degree in bioengineering from the Georgia Institute of Technology, Atlanta, in 2004.

From 2005 to 2006, he was with Mindray Medical, a medical device company based in Shenzhen, China. In 2006, he joined Siemens Corporate Technology, Beijing, China, as a Research Scientist. His current research interests include ultrasound imaging and validation methods for medical image processing.

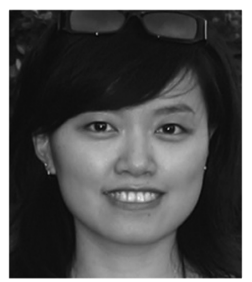

Yan Yang received the B.S degree from the Department of Biomedical Engineering, Tsinghua University, Beijing, China, in 2002. She is currently pursuing the Ph.D. degree at the Georgia Institute of Technology, Atlanta.

Her research interests include medical imaging, image processing, and computer vision.

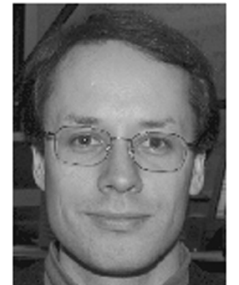

Steven Haker received the Ph.D. degree in control and dynamical systems from the University of Minnesota, Minneapolis, in 1999.

He is presently a Research Scientist at the Surgical Planning Laboratory of the Brigham and Women's Hospital of Harvard Medical School, Boson, MA. His research interests are in medical imaging, image processing, and computer vision.

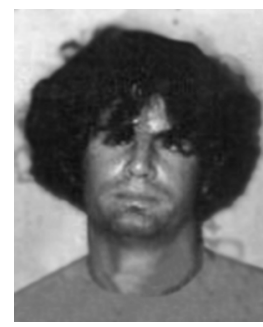

Allen Tannenbaum was born in New York City in 1953. He received the Ph.D. degree in mathematics from Harvard University, Cambridge, MA, in 1976.

$\mathrm{He}$ has held faculty positions at the Weizmann Institute of Science; McGill University, Montréal, QC, Canada; ETH, Zurich, Switzerland; the Technion-Israel Institute of Technology, Haifa; the Ben-Gurion University of the Negev, Israel; and the University of Minnesota, Minneapolis. He is presently the Julian Hightower Professor of Electrical and Biomedical Engineering at The Georgia Institute of Technology, Atlanta/Emory. He has done research in image processing, medical imaging, computer vision, robust control, systems theory, robotics, semiconductor process control, operator theory, functional analysis, cryptography, algebraic geometry, and invariant theory. 